\title{
Decay of correlation functions in hard-sphere mixtures: Structural crossover
}

\author{
C. Grodon \\ Max-Planck-Institut für Metallforschung, Heisenbergstrasse 3, D-70569 Stuttgart, Germany \\ and Institut für Theoretische und Angewandte Physik, Universität Stuttgart, Pfaffenwaldring 57, \\ D-70569 Stuttgart, Germany \\ M. Dijkstra \\ Debye Institute, Soft Condensed Matter Group, Utrecht University, Princetonplein 5, 3584 CC Utrecht, \\ The Netherlands \\ R. Evans \\ H.H. Wills Physics Laboratory, University of Bristol, Bristol BS8 1TL, United Kingdom; \\ Max-Planck-Institut für Metallforschung, Heisenbergstrasse 3, D-70569 Stuttgart, Germany \\ and Institut für Theoretische und Angewandte Physik, Universität Stuttgart, Pfaffenwaldring 57, \\ D-70569 Stuttgart, Germany \\ R. Roth \\ Max-Planck-Institut für Metallforschung, Heisenbergstrasse 3, D-70569 Stuttgart, Germany \\ and Institut für Theoretische und Angewandte Physik, Universität Stuttgart, Pfaffenwaldring 57, \\ D-70569 Stuttgart, Germany
}

(Received 23 June 2004; accepted 2 August 2004)

\begin{abstract}
We investigate the decay of pair correlation functions in a homogeneous (bulk) binary mixture of hard spheres. At a given state point the asymptotic decay $r \rightarrow \infty$ of all three correlation functions is governed by a common exponential decay length and a common wavelength of oscillations. Provided the mixture is sufficiently asymmetric, size ratios $q \lesssim 0.7$, we find that the common wavelength reflects either the size of the small or that of the big spheres. By analyzing the (complex) poles of the partial structure factors we find a sharp structural crossover line in the phase diagram. On one side of this line the common wavelength is approximately the diameter of the smaller sized spheres whereas on the other side it is approximately the diameter of the bigger ones; the wavelength of the longest ranged oscillations changes discontinuously at the structural crossover line. Using density functional theory and Monte Carlo simulations we show that structural crossover also manifests itself in the intermediate range behavior of the pair correlation functions and we comment on the relevance of this observation for real (colloidal) mixtures. In highly asymmetric mixtures, $q \leqslant 0.1$, where there is metastable fluid-fluid transition, we find a Fisher-Widom line with two branches. This line separates a region of the phase diagram where the decay of pair correlations is oscillatory from one in which it is monotonic. (C) 2004 American Institute of Physics.
\end{abstract}

[DOI: $10.1063 / 1.1798057$ ]

\section{INTRODUCTION}

The total correlation functions $h_{i j}(r)$, where $i$ and $j$ label species, or equivalently the radial (pair) distribution functions $g_{i j}(r) \equiv h_{i j}(r)+1$, play a central role in understanding the equilibrium structure of homogeneous fluid mixtures. The radial distribution functions determine the probability of finding a particle of component $j$ at a distance $r$ from another particle of component $i$. These quantities can be calculated within different theoretical frameworks, i.e., via computer simulation, integral equation theories based on the OrnsteinZernike equations with suitable closure relations, or density functional theory using the so-called test particle route. ${ }^{1}$ In neutron diffraction experiments, using isotopic substitution, the partial structure factors can be measured. ${ }^{2}$ These are, essentially, the Fourier transforms $\hat{h}_{i j}(k)$ and the total correlation functions of the mixture can, in principle, be recovered by an inverse Fourier transform. However, obtaining detailed information about $h_{i j}(r)$ at large separations requires accurate data through the full range of wave numbers $k$, and this is difficult to obtain from scattering experiments. On the other hand, recent advances in experimental techniques now permit the accurate determination of the pair correlation function for a two-dimensional colloidal system in real space using video microscopy; see, for example, Refs. 3, 4.

Despite its importance in the description of fluid mixtures, relatively little is known about the generic properties of $g_{i j}(r)$. Most of the studies concerned with pair correlation functions concentrate on the behavior of $g_{i j}(r)$ at small separations $r$, which provides information about the distribution of nearest and next nearest neighbors. Clearly, the shortranged behavior depends strongly on local details of the interparticle interactions, i.e., on the chemistry. By contrast, at intermediate and large separations one might expect that such details are less important and it should be possible to make some general predictions. Indeed, this is the case. For 
example, for a pure fluid with short-ranged interactions (interparticle potentials of finite support or decaying exponentially) it can be shown ${ }^{5-7}$ that the function $r g(r)$ will decay to its asymptotic value of 1 in only two possible ways: (i) purely exponential, i.e., monotonically or (ii) exponentially damped oscillatory. The characteristic decay length and wavelength of the oscillations depend on the thermodynamic state point of the fluid. For fluids with attractive interaction potentials which exhibit liquid-gas coexistence there is a line in the phase diagram where the ultimate decay of $r h(r)$ crosses over from monotonic to oscillatory. ${ }^{7}$ Such a line appears to be a general feature, ${ }^{8,9}$ it was first proposed by Fisher and Widom. ${ }^{10}$

In this paper we focus on understanding the intermediate and asymptotic decay in binary mixtures. For fluid mixtures in which the interparticle potentials are short-ranged, analysis of the mixture Ornstein-Zernike equations leads to an important general prediction, ${ }^{6}$ namely, that all the total correlation functions $r h_{i j}(r)$ must decay to zero as $r \rightarrow \infty$, with a common exponential decay length and a common wavelength, assuming one is in the oscillatory part of the phase diagram. Evans et al. ${ }^{6}$ went on to demonstrate that the general prediction for the leading order asymptotic form [see Eq. (7) below] remains accurate down to surprisingly small separations, i.e., down to the second maximum in $h_{i j}(r)$, for a binary hard-sphere mixture with size ratio $q=0.5$, treated in Percus-Yevick approximation. Here we revisit the additive binary hard-sphere mixture and enquire about the nature of pairwise correlations as the packing fractions of big and small spheres are varied for a wide range of size ratios. We concentrate on the additive hard-sphere mixture since for this system well-established and well-tested theoretical tools are readily available. Moreover, the hard-sphere mixture can be viewed as a generic reference system for a fluid mixture (with short-ranged interactions). Thus our results should shed light on the decay of pair correlations in a much wider class of liquid mixtures.

Our paper is organized as follows. In Sec. II we start by summarizing the basic concepts of the theory of asymptotic decay in binary fluid mixtures. We present two different approaches. First, the direct binary mixture route, which we apply for weakly asymmetric mixtures; and second, the effective one-component route, which we apply in strongly asymmetric cases, $q \ll 1$. The second route corresponds to integrating out the degrees of freedom of the small spheres to obtain an effective pair potential between two big spheresthe so-called depletion potential. In Sec. III we present the results of our calculations. By calculating the complex poles of $\hat{h}_{i j}(k)$ using two different approximations for the pair direct correlation functions we find a new structural crossover line in the phase diagram. On one side of this line the common wavelength of oscillations in $h_{i j}(r)$ is set by the diameter of the smaller spheres whereas on the other side this is set by the diameter of the larger spheres. Using density functional theory and the test particle procedure we determine $h_{i j}(r)$ at intermediate values of $r$ for states on opposite sides of the line. There are dramatic differences in $h_{i j}(r)$ that are confirmed by results of Monte Carlo simulations for $q$ $=0.5$. For the highly asymmetric cases, $q=0.1$ and 0.05 , we find a fluid-fluid spinodal accompanied by a Fisher-Widom (FW) line with two branches; the transition lines all lie within the metastable (with respect to crystallization) region of the phase diagram. We conclude with a discussion in Sec. IV.

\section{THEORY OF ASYMPTOTIC DECAY OF CORRELATIONS IN BINARY MIXTURES}

We follow the general approach of Refs. 6, 7 and study the asymptotic decay of the total correlation functions $h_{i j}(r)$ in a bulk binary hard-sphere mixture. The system is described fully by the number densities $\rho_{i}, i=s, b$, of the small (radius $R_{s}$ ) and the big (radius $R_{b}$ ) spheres, respectively, and the size ratio $q=R_{s} / R_{b}$. In modeling binary atomic mixtures the size ratio $q$ would usually be close to 1 . Here, however, we have in mind mainly mixtures of spherical colloids for which the radius of each component can vary in a huge range, from several nanometers to a few micrometers. Hence a size ratio $q \ll 1$ is easily achieved. Depending on the value of $q$, we might choose to study the asymptotic behavior using different theoretical frameworks.

For relatively symmetric mixtures, say $q \gtrsim 0.2$, an approach which treats both components on equal footing is most appropriate. As we shall see, this binary mixture route requires a theory that can predict accurately partial pair direct correlation functions $c_{i j}^{(2)}(r)$ with $i, j=s, b$. Here we use density functionals based on the fundamental measure theory (FMT) for hard-sphere mixtures ${ }^{11-13}$ to generate $c_{i j}^{(2)}(r)$. In the case of the original Rosenfeld functional ${ }^{11}$ the pair direct correlation functions obtained from this route are exactly those of Percus-Yevick theory, ${ }^{14}$ while for the White Bear version of FMT $^{12,13}$ slightly different pair direct correlation functions result.

If the size ratio $q<0.2$, the binary mixture route becomes less reliable since standard closure approximations to integral equation theories exhibit failings for asymmetric mixtures. This can be seen by noting that the metastable fluid-fluid phase separation found in simulations for $q \leqslant 0.1$ is completely absent in the Percus-Yevick treatment of the binary additive hard-sphere mixture; ${ }^{15}$ this does not predict a spinodal. ${ }^{16}$ For small values of $q$ the binary mixture can be mapped onto an effective one-component fluid of big particles that interact with each other via a pairwise effective (depletion) potential. ${ }^{17}$ Although formally an infinite number of many-body interactions between big particles result from the exact mapping, ${ }^{15}$ an explicit calculation of the three-body interaction $^{18}$ for $q=0.2$ found this to be small, implying that for $q<0.2$ an accurate description of the big-big $(b b)$ correlations and of thermodynamics in the binary mixture should be given by an effective Hamiltonian for the bulk onecomponent fluid which is based on only the pairwise depletion potential plus structure independent contributions. ${ }^{15}$

Below we describe both routes to $b b$ pair correlations in the binary hard-sphere mixture.

\section{A. Binary mixture route}

In the bulk mixture the total correlation functions $h_{i j}(r)$ are related to the pair direct correlation functions $c_{i j}^{(2)}(r)$ via the following mixture Ornstein-Zernike (OZ) equations: 


$$
h_{i j}\left(r_{12}\right)=c_{i j}^{(2)}\left(r_{12}\right)+\sum_{k=s, b} \rho_{k} \int d^{3} r_{3} c_{i k}^{(2)}\left(r_{13}\right) h_{k j}\left(r_{32}\right),
$$

with $r_{i j}=\left|\mathbf{r}_{i}-\mathbf{r}_{j}\right|$. These equations can be considered as defining the pair direct correlation functions. If an additional relation between $h_{i j}(r)$ and $c_{i j}^{(2)}(r)$, a so-called closure relation, is specified, then the $\mathrm{OZ}$ equations can be solved to yield explicit correlation functions. Alternatively, pair direct correlation functions obtained from a different theory can be inputted into Eq. (1). In the present binary mixture route, we employ a density functional for the hard-sphere mixture to generate the pair direct correlation functions. Asymptotic analysis based on Eq. (1) then leads to predictions for $h_{i j}(r)$ as $r \rightarrow \infty$. We then compare these predictions with results of numerical calculations of the total correlation functions obtained from the minimization of the same density functional using the test-particle procedure.

Assuming that the direct correlation functions are given, the set of coupled equations (1) can be solved formally in Fourier space, and the solution is written as

$$
\hat{h}_{i j}(k)=\frac{\hat{N}_{i j}(k)}{\hat{D}(k)},
$$

where $\hat{h}_{i j}(k)$ is the three-dimensional Fourier transform of $h_{i j}(r)$. Note that the numerators of Eq. (2) depend on the indices $i$ and $j$, and are given by

$$
\begin{aligned}
& \hat{N}_{s s}(k)=\hat{c}_{s s}^{(2)}(k)+\rho_{b}\left[\hat{c}_{b s}^{(2)}(k)^{2}-\hat{c}_{s s}^{(2)}(k) \hat{c}_{b b}^{(2)}(k)\right], \\
& \hat{N}_{b b}(k)=\hat{c}_{b b}^{(2)}(k)+\rho_{s}\left[\hat{c}_{b s}^{(2)}(k)^{2}-\hat{c}_{s s}^{(2)}(k) \hat{c}_{b b}^{(2)}(k)\right], \\
& \hat{N}_{b s}(k)=\hat{c}_{b s}^{(2)}(k)=\hat{c}_{s b}^{(2)}(k)=\hat{N}_{s b}(k),
\end{aligned}
$$

while the denominator of Eq. (2) is common to all three equations and can be written as

$$
\hat{D}(k)=\left[1-\rho_{s} \hat{c}_{s s}^{(2)}(k)\right]\left[1-\rho_{b} \hat{c}_{b b}^{(2)}(k)\right]-\rho_{s} \rho_{b} \hat{c}_{b s}^{(2)}(k)^{2} .
$$

From the inverse Fourier transform we can obtain the total correlation function in real space:

$$
r h_{i j}(r)=\frac{1}{2 \pi^{2}} \int_{0}^{\infty} d k k \sin (k r) \hat{h}_{i j}(k) .
$$

Henceforward we assume the singularities of $\hat{h}_{i j}(k)$ to be (simple) poles. ${ }^{6}$ If we denote the $n$th pole of $\hat{h}_{i j}(k)$, i.e., the $n$th solution of the equation $\hat{D}(k)=0$ in the upper complex $k$ half plane by $p_{n}$, and the corresponding residue of $k \hat{h}_{i j}(k)$ by $R_{n}^{i j}$, then we can perform the inverse Fourier transform via the residue theorem and write the total correlation function as ${ }^{6}$

$$
r h_{i j}(r)=\frac{1}{2 \pi} \sum_{n} R_{n}^{i j} e^{i p_{n} r} .
$$

Each pole contributes to the total correlation function either a pure exponential term, if $p_{n}$ is purely imaginary, or an exponential damped cosine, if $p_{n}$ is complex. Accounting for the behavior of $h_{i j}(r)$ for small values of $r$ requires contributions from several (in general an infinite number) poles, while it is clear that in the asymptotic regime, $r \rightarrow \infty$, the pole that gives rise to the slowest exponential decay will dominate, i.e., the pole with the smallest imaginary part. We call this pole the leading order pole: $p=a_{1}+i a_{0}$.

So far we did not specify the direct correlation functions $c_{i j}^{(2)}(r)$ and the argument holds for all fluid mixtures with short-ranged interparticle potentials giving rise to simple poles. ${ }^{6}$ However, from the structure of the mixture OZ equations (2) we can ascertain that all three total correlation functions have a common asymptotic decay of the form

$$
h_{i j}(r) \sim \frac{A_{i j}}{r} \exp \left(-a_{0} r\right) \cos \left(a_{1} r-\Theta_{i j}\right), \quad r \rightarrow \infty,
$$

with a common characteristic decay length $a_{0}^{-1}$ and wavelength of oscillations $2 \pi / a_{1}$. Only the amplitudes $A_{i j}$ and phases $\Theta_{i j}$ depend on the indices $i j$. (The results generalize straightforwardly to a multicomponent mixture.) This conclusion is remarkable because the two basic length scales in the problem, namely, the radii or diameters of the particles, can be very different. It is clear that if we consider a binary mixture in which the concentration of the small particles is high, while that of the big ones is low, then the length scale of the common asymptotic decay of all three total correlation functions should be set by the size of the small particles. ${ }^{17}$ On the other hand, we could consider the opposite case in which the concentration of the big spheres is high, while that of the small ones is low and conclude that the length of the common asymptotic decay is set by the size of the big particles. It is less clear what the asymptotic decay of the binary mixture should be in the case where the two components have similar densities. Nevertheless, if the radii of the components are comparable, i.e. $q \sim 1$, the system will have no problem to find a common asymptotic length scale, which should interpolate smoothly between the basic length scales. However, if the radius of the small spheres is significantly smaller than that of the big ones, little is established about the nature of the asymptotics, in particular its variation with concentration, and it is this topic we address here.

\section{Pair direct correlation functions}

In order to determine the asymptotics we must calculate the zeros of $\hat{D}(k)$-see Eqs. (2) and (4)—which requires, in turn, $\hat{c}_{i j}^{(2)}(k)$. Since we are interested in a comparison between asymptotic results from the OZ equations (1) and those obtained numerically from the test particle route within the framework of density functional theory (DFT), we choose to use the excess (over ideal gas) Helmholtz free energy functional $\mathcal{F}_{\text {ex }}\left[\left\{\rho_{i}\right\}\right]$ to generate the pair direct correlation functions. Within DFT we have ${ }^{19}$

$$
c_{i j}^{(2)}\left(r_{12}\right)=-\left.\beta \frac{\delta^{2} \mathcal{F}_{\text {ex }}\left[\left\{\rho_{i}\right\}\right]}{\delta \rho_{i}\left(\mathbf{r}_{1}\right) \delta \rho_{j}\left(\mathbf{r}_{2}\right)}\right|_{\rho_{i}\left(\mathbf{r}_{1}\right)=\rho_{i}, \rho_{j}\left(\mathbf{r}_{2}\right)=\rho_{j}} .
$$

For the system of interest, namely binary mixtures of hard spheres, we employ Rosenfeld's successful FMT in two different formulations: (i) the original Rosenfeld functional, ${ }^{11}$ which generates the well-known Percus-Yevick pair direct correlation functions, ${ }^{14}$ and (ii) the White Bear version, ${ }^{12,13}$ which modifies the FMT so that the underlying bulk equation 
of state is that due to Mansoori-Carnahan-Starling-Leland (MCSL). ${ }^{20,21}$ The MCSL equation of state is closer to that found in computer simulations than is the Percus-Yevick compressibility equation of state which underlies the original FMT.

The structure of the FMT functionals is given by ${ }^{11-13}$

$$
\beta \mathcal{F}_{\text {ex }}\left[\left\{\rho_{i}\right\}\right]=\int d^{3} r^{\prime} \Phi\left(\left\{n_{a}\left(\mathbf{r}^{\prime}\right)\right\}\right),
$$

i.e., the excess free energy density $\beta^{-1} \Phi$, with $\beta$ $=\left(k_{B} T\right)^{-1}$, is a function of weighted densities of the $\nu$-component mixture that have the form

$$
n_{\alpha}(\mathbf{r})=\sum_{i=1}^{\nu} \int d^{3} r^{\prime} \rho_{i}\left(\mathbf{r}-\mathbf{r}^{\prime}\right) \omega_{\alpha}^{i}\left(\mathbf{r}^{\prime}\right)
$$

The weight functions $\omega_{\alpha}^{i}$ describe the fundamental geometrical measures of a sphere of component $i{ }^{11}$ There are four scalar and two vector weights which are labeled by $\alpha$ (see the Appendix). Within FMT the pair direct correlation function takes the form

$$
c_{i j}^{(2)}(r)=-\sum_{\alpha, \beta} \frac{\partial^{2} \Phi}{\partial n_{\alpha} \partial n_{\beta}} \omega_{\alpha}^{i} \otimes \omega_{\beta}^{j},
$$

with $\otimes$ denoting the convolution product. The convolutions of weight functions in Eq. (11) are the same for both versions of FMT and the results are given in the Appendix. The second partial derivatives of $\Phi$ with respect to the weighted densities $n_{\alpha}$ depend on the particular version of FMT. In the case of the Rosenfeld functional, ${ }^{11}$ Eq. (11) recovers precisely the Percus-Yevick pair direct correlation functions, ${ }^{14}$ which therefore require no further discussion. For the White Bear version the pair direct correlation function of the pure fluid was given explicitly in Refs. 12, 13. The general form is similar to that of the Percus-Yevick (PY) pair direct correlation function. However, the results for $c^{(2)}(r)$ from the White Bear version are slightly more accurate when compared to results of computer simulations.

For the binary mixture case, we find the pair direct correlation functions obtained from the White Bear version are again similar to those corresponding to the PY closure (see the Appendix). However, the two approaches are sufficiently different for us to test whether the results for asymptotics depend sensitively on the approximations introduced by the use of approximate pair direct correlation functions. We show an example of the pair direct correlation functions obtained from both functionals in Fig. 1, for a binary hardsphere mixture with $q=0.4, \eta_{b}=0.1$, and $\eta_{s}=0.1$, where the packing fraction $\eta_{i}=(4 \pi / 3) R_{i}^{3} \rho_{i}$. The overall agreement between the two approaches is good; differences can hardly be seen, on the scale of this plot, for $c_{s s}^{(2)}(r)$ and for $c_{b s}^{(2)}(r) \equiv c_{s b}^{(2)}(r)$. For this choice of parameters, small differences in $c_{b b}^{(2)}(r)$ are visible.

Since the Fourier transforms $\hat{c}_{i j}^{(2)}(k)$ are given analytically in both the Rosenfeld and White Bear schemes, the poles can be determined directly by solving $\hat{D}(k)=0 ; k$ complex, where $\hat{D}(k)$ is given by Eq. (4).

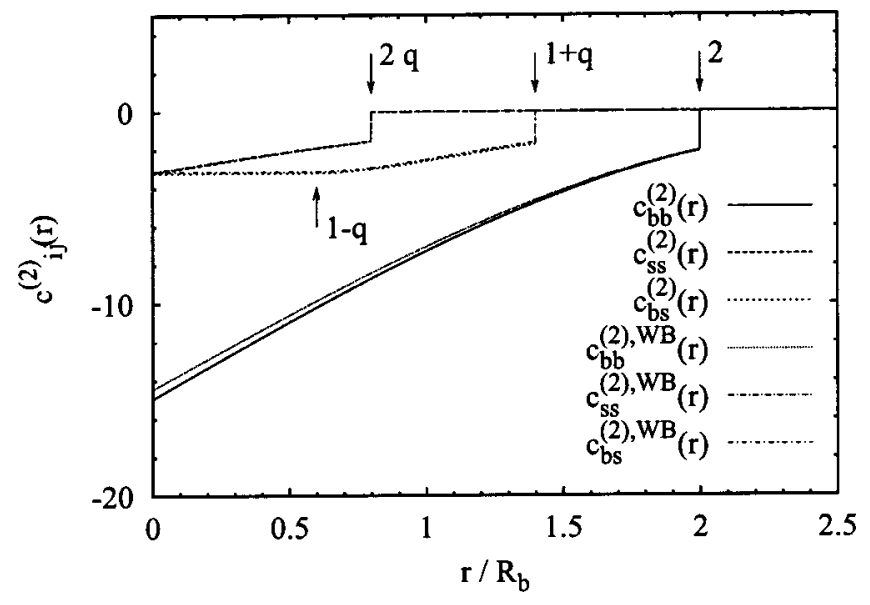

FIG. 1. The pair direct correlation functions $c_{i j}^{(2)}(r)$ obtained from both the Rosenfeld DFT [equivalent to Percus-Yevick theory (Ref. 14)] and the White Bear version for a hard-sphere mixture with packing fractions $\eta_{b}$ $=0.1$ and $\eta_{s}=0.1$ and size ratio $q \equiv R_{s} / R_{b}=0.4$. Whereas the results for small-small $(s s)$ and big-small ( $b s)$ correlations are almost indistinguishable between the two theories, there are visible differences between the results for big-big $(b b)$ correlations.

\section{B. Effective one-component route}

For asymmetric mixtures with size ratios $q \leqslant 0.1$, it was found, in Monte Carlo simulations of the effective onecomponent fluid, ${ }^{15}$ that the binary hard-sphere liquid can exhibit fluid-fluid phase separation, although the latter is always metastable with respect to the fluid-solid phase transition. As mentioned earlier, this feature is not captured by the standard Percus-Yevick closure of the mixture OZ equations, and, hence points to possible failings of integral equation closures for highly asymmetric mixtures-see, e.g., Ref. 15 and references therein. Since the presence of fluidfluid phase separation should be accompanied by a so-called Fisher-Widom line, ${ }^{7,10}$ at which the asymptotic behavior of bulk pair correlations changes from damped oscillatory to purely exponential decay, this failure of the conventional binary route to account for a spinodal also has implications for the asymptotics of correlations.

In order to determine the asymptotic behavior of correlations for asymmetric mixtures we change from a description of the full binary mixture and adopt an effective onecomponent viewpoint. To this end we integrate out the degrees of freedom of the small particles and thereby map their influence in terms of an effective Hamiltonian, ${ }^{15,22}$

$$
\mathcal{H}_{\text {eff }}=\mathcal{H}_{b b}+\Omega,
$$

where $\mathcal{H}_{b b}$ describes the direct interactions between big particles and $\Omega=\Omega_{0}+\Omega_{1}+\Omega_{2}+\cdots$ is the grand potential of the sea of small particles in the presence of a fixed configuration of big ones. $\Omega_{0}$ is the zero-body term, $-p_{s} V$, i.e., the grand potential of a homogeneous sea of small particles of volume $V$ and pressure $p_{s} . \Omega_{1}$, the one-body term, is $N_{b} \omega_{1}$, where $\omega_{1}$ is the excess chemical potential of species $b$ at infinite dilution, i.e., the difference in grand potential between the sea of small particles with and without a single big particle. $N_{b}$ is the number of big particles. $\Omega_{2}$ is the contribution from the effective interaction between pairs of big 
particles. ${ }^{15,17,23}$ Higher order terms $\Omega_{3}, \Omega_{4}, \ldots$ take into account effective many-body interactions between three, four, and so forth, big particles. However, for the size ratios of interest here, these are expected to be small. ${ }^{18}$ Thus, in the present context we truncate the effective Hamiltonian after $\Omega_{2}$. For the calculations of the asymptotic decay of the effective one-component fluid the structure independent terms $\Omega_{0}$ and $\Omega_{1}$, which depend upon only the chemical potential $\mu_{s}$ of the reservoir of small particles, are irrelevant and, for our present study, the mapping of the hard-sphere mixture onto an effective one-component fluid is characterized by the effective interaction potential between two big particles,

$$
\Phi_{b b}^{\mathrm{eff}}(r)=\Phi_{b b}^{\mathrm{HS}}(r)+W(r)
$$

i.e., the sum of the bare hard-sphere interaction potential $\Phi_{b b}^{\mathrm{HS}}(r)$ and the depletion potential $W(r)$. For $W(r)$, which depends on $\mu_{s}$, we use the parameterized form from Ref. 17, which enforces the correct asymptotic behavior of the depletion potential. Note that the asymptotic behavior of the depletion potential determines that of the big-big correlation function in the limit of vanishing density of the big particles, $\rho_{b} \rightarrow 0$, since

$$
\lim _{\rho_{b} \rightarrow 0} h_{b b}(r)=\exp \left[-\beta \Phi_{b b}^{\mathrm{eff}}(r)\right]-1 .
$$

In this limit the asymptotic decay of $h_{b b}(r)$ is given by Eq. (7), with $a_{0}$ and $a_{1}$ determined by the zeros of [1 $\left.-\rho_{s} \hat{c}_{s s}(k)\right]$. $^{17}$

Within the effective one-component picture we use the one-component $\mathrm{OZ}$ equation which relates the effective total correlation function $h_{\text {eff }}(r)$ to the effective pair direct correlation function $c_{\mathrm{eff}}^{(2)}(r)$ of a fluid interacting via the potential (13). The one-component OZ equation is, of course, much simpler in structure than its mixture counterpart, Eq. (1), and in Fourier space is given by

$$
\hat{h}_{\mathrm{eff}}(k)=\frac{\hat{c}_{\mathrm{eff}}^{(2)}(k)}{1-\rho_{b} \hat{c}_{\mathrm{eff}}^{(2)}(k)} .
$$

In order to ascertain the asymptotic behavior of $h_{\text {eff }}(r)$, which is equivalent to $h_{b b}(r)$ of the binary mixture, we must now determine the pole structure of Eq. (15) by finding solutions $p_{n}$ of the equation

$$
1-\rho_{b} \hat{c}_{\text {eff }}^{(2)}\left(p_{n}\right)=0 .
$$

This approach is quite distinct from the full binary mixture treatment, because we can no longer input an analytic expression for the pair direct correlation function. Rather we must solve the OZ equation (15) via a closure relation. We apply either the PY or the hypernetted-chain (HNC) closure and obtain a numerical solution for $c_{\mathrm{eff}}^{(2)}(r)$. The solution of Eq. (16) can then be determined numerically using the procedure described in Refs. 7-9.

Another important difference between the binary route and the effective one-component route is that we describe the full binary mixture in the canonical ensemble, whereas the other route is most efficiently implemented in the semigrand-canonical ensemble in which the big spheres are treated canonically, while the small spheres are coupled to a reservoir at fixed chemical potential $\mu_{s}$. The integrating out procedure then implies that the depletion potential $W(r)$ between big particles depends on the reservoir density of small spheres, $\rho_{s}^{r}$, rather than on its system counterpart, $\rho_{s}$. A relation between the system packing fraction $\eta_{s}$ and the reservoir value $\eta_{s}^{r}=4 \pi R_{s}^{3} \rho_{s}^{r} / 3$ can be obtained from the effective Hamiltonian. ${ }^{15,22}$ From the effective Hamiltonian based on the Rosenfeld functional (equivalent to PY compressibility route) an accurate conversion formula is given by ${ }^{22}$

$$
\begin{aligned}
\eta_{s}= & \left(1-\eta_{b}\right) \eta_{s}^{r}-3 q \eta_{b} \eta_{s}^{r} \frac{1-\eta_{s}^{r}}{1+2 \eta_{s}^{r}}-3 q^{2} \eta_{b} \eta_{s}^{r} \frac{\left(1-\eta_{s}^{r}\right)^{2}}{\left(1+2 \eta_{s}^{r}\right)^{2}} \\
& -q^{3} \eta_{b} \eta_{s}^{r} \frac{\left(1-\eta_{s}^{r}\right)^{3}}{\left(1+2 \eta_{s}^{r}\right)^{2}} .
\end{aligned}
$$

The resulting conversions yield results that are in very good agreement with those of Monte Carlo simulations for size ratios $q=0.1$ and 0.05 , and a wide range of values of $\eta_{b}$-see Fig. 3 of Ref. 22. Similarly, one can derive the corresponding conversion formula based on an effective Hamiltonian obtained from the White Bear version of FMT. However, for the size ratios considered here, the difference between the numerical results is negligibly small, and we have chosen to employ Eq. (17) in our conversions.

\section{RESULTS OF CALCULATIONS}

\section{A. Pure hard-sphere fluid}

We begin by recalling briefly results for the pole structure in the well-studied pure hard-sphere fluid. ${ }^{9,17,24-26}$ This system plays an important role as the limiting case of a binary mixture in which either the size ratio is close to unity or the density of one component goes to zero. The trajectories of poles in the pure fluid are shown in Fig. 2(a). The poles are indicated by crosses for a packing fraction of $\eta=0.1$ and $\pi_{i}$ denotes the pole $i$ with $i=1,2, \ldots$. In the pure case the pole $\pi_{1}$ has, for all packing fractions $\eta$, the smallest imaginary part, and therefore it is always the leading order pole. It describes, as discussed already, the asymptotic behavior of the total correlation function. Other poles are ordered by increasing imaginary part $a_{0}$ and are denoted $\pi_{2}, \pi_{3}$, and so on.

When $\eta$ is changed all the poles move along the trajectories shown in such a way that the order of the poles remains unchanged. As $\eta$ is decreased, the density-density correlation length $\xi$ in the fluid must decrease. This corresponds to an increase in $a_{0}\left(\pi_{1}\right) \equiv \xi^{-1}$. For an increase of $\eta$, correlations decay more slowly, which corresponds to a decrease in $a_{0}\left(\pi_{1}\right)$. Note that in the limit $\eta \rightarrow 1$ the imaginary part of all the poles vanishes at least in the framework of PY theory. ${ }^{26}$ In the present context we are interested solely in the equilibrium fluid phase and in order to avoid the complications of freezing we shall restrict the (total) packing fraction to $\eta<0.494$, the freezing value for pure hard spheres. ${ }^{27}$ Note that close packing of hard spheres in dimension $d=3$ occurs at $\eta=\sqrt{2} \pi / 6 \approx 0.7405$.

If a binary mixture is considered in which either the packing fraction of one component is very small or the size 

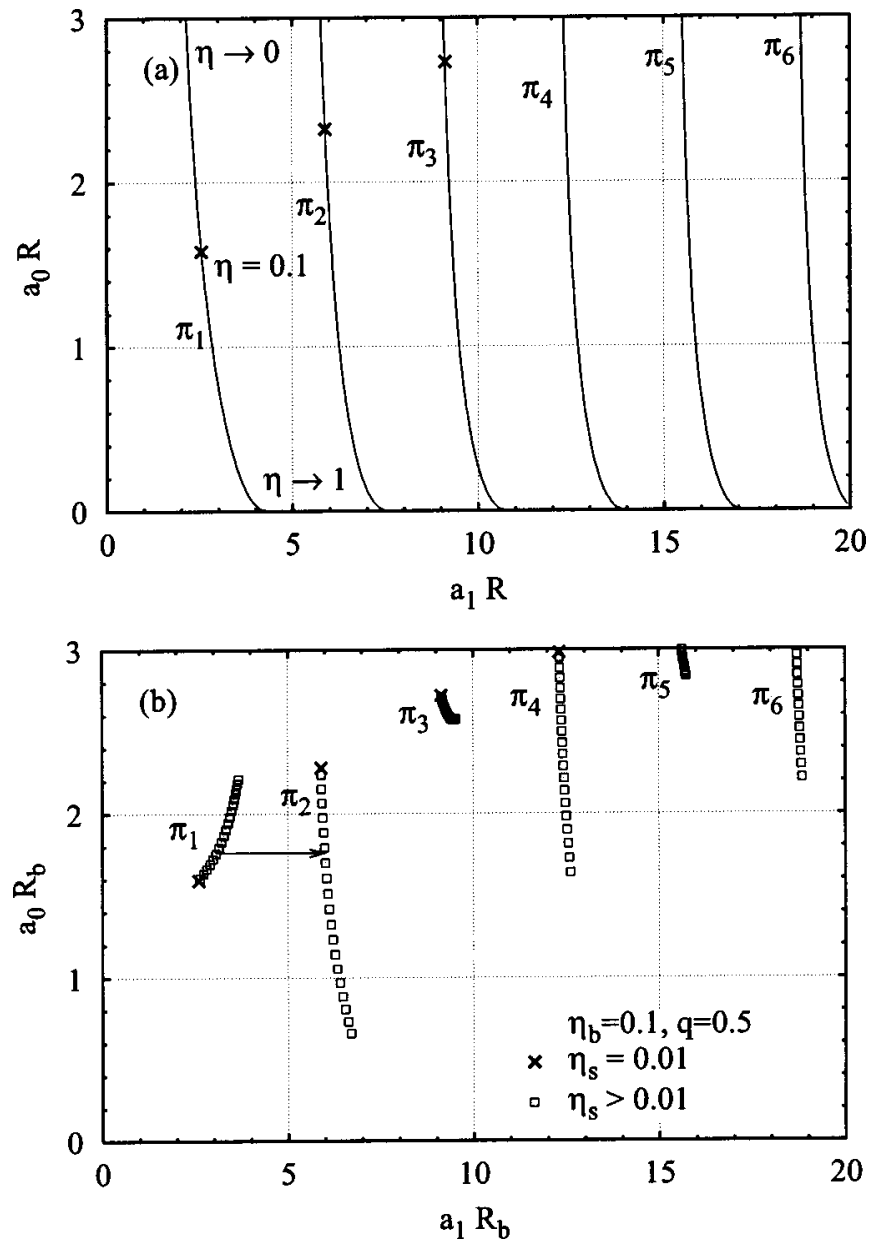

FIG. 2. (a) The trajectories of poles for a one-component fluid of hard spheres of radius $R$ treated in the Percus-Yevick approximation. The crosses denote poles evaluated for a packing fraction $\eta=0.1 . a_{0}$ denotes the imaginary and $a_{1}$ the real part of each pole. Upon decreasing $\eta$, each pole $\left(\pi_{1}\right.$, $\pi_{2}$, etc.) shifts to higher values of $a_{0}$ but the sequence remains the same, i.e., $\pi_{1}$ has the smallest imaginary part. In the high density limit, $\eta \rightarrow 1$, the imaginary part of each pole vanishes. (b) Trajectories for a binary hardsphere mixture with size ratio $q \equiv R_{s} / R_{b}=0.5$ obtained from Percus-Yevick theory. The packing fraction of the big spheres is fixed at $\eta_{b}=0.1$. The crosses denote poles for a small sphere packing fraction $\eta_{s}=0.01$. The squares indicate trajectories for increasing values of $\eta_{s}$. The imaginary part of $\pi_{1}$ increases with $\eta_{s}$ while that of $\pi_{2}$ decreases, leading to crossover, see the horizontal arrow at $\eta_{s}^{*}=0.126 . a_{1}$ increases by a factor of about $1 / q$ $=2$ at the crossover.

ratio $q$ is close to unity, then the pole structure of the mixture should constitute a small perturbation around the pole structure of the pure fluid. We find that in these limiting cases all poles move in the same direction in the complex plane upon changing parameters and the leading order pole remains $\pi_{1}$. However, as we shall see below, the trajectories do alter when higher packing of the minority component or more asymmetric mixtures are considered.

\section{B. Weakly asymmetric binary mixtures}

Here we consider binary mixtures with $q>0.2$ and arbitrary packing fractions of the components, and we apply the binary mixture route to determine the pole structure and the leading order pole. We start by considering a mixture with $q=0.5$ and packing fractions $\eta_{s}=0.01$ and $\eta_{b}=0.1$; the cor- responding results are shown as crosses in Fig. 2(b). As we increase the packing fraction of the small spheres $\eta_{s}$ while keeping $\eta_{b}$ fixed, the imaginary part of $\pi_{1}$ increases while that of $\pi_{2}$ decreases, as is shown by the open symbols in Fig. 2(b). This behavior of $\pi_{1}$ is opposite from what is found in the pure case and gives rise to the effect that for a certain value of $\eta_{s}$, which we denote $\eta_{s}^{*}(=0.126)$, the imaginary part of $\pi_{1}$ and of $\pi_{2}$ are identical and, hence, the asymptotic behavior of the total correlation functions is determined by two poles with the same $a_{0}$ but different $a_{1}$. If $\eta_{s}$ is increased further the role of the poles $\pi_{1}$ and $\pi_{2}$ is interchanged and the leading-order pole is then $\pi_{2}$. This crossover from one leading-order pole to another, with distinct real parts, is indicated by an arrow in Fig. 2(b). When the crossover occurs the wavelength of oscillations of the three total correlation functions, in the asymptotic regime, jumps from a value that is set by the diameter of the big spheres at low $\eta_{s}$ to one that is set by the diameter of the small ones at higher $\eta_{s}$, and hence changes by roughly a factor of $q$ (equal to 0.5 in the present case). Close to the crossover point, $\eta_{s}^{*}$, there are two poles which have similar imaginary parts, $a_{0}$ and $\tilde{a}_{0}$, and which will both contribute to the exponentially damped oscillatory decay of the total correlation functions in the intermediate regime, provided that the amplitudes, $A_{i j}$ and $\widetilde{A}_{i j}$, of both contributions are of comparable size, i.e., we expect

$$
\begin{aligned}
r h_{i j}(r) \sim & A_{i j} \exp \left(-a_{0} r\right) \cos \left(a_{1} r-\Theta_{i j}\right)+\tilde{A}_{i j} \\
& \times \exp \left(-\widetilde{a}_{0} r\right) \cos \left(\tilde{a}_{1} r-\widetilde{\Theta}_{i j}\right), \quad r \rightarrow \infty,
\end{aligned}
$$

where the first contribution corresponds to $\pi_{1}$, with $a_{1}$ $\sim \pi / R_{b}$, and the second to $\pi_{2}$, with $\tilde{a}_{1} \sim \pi /\left(q R_{b}\right)$. At the crossover point $a_{0}=\tilde{a}_{0}$. Note that the higher order poles $\pi_{3}$, $\pi_{4}$, etc. play no role in determining the asymptotics for $q$ $=0.5$.

For a slightly more asymmetric binary mixture, with $q$ $=0.35$, the crossover scenario is more complicated. In Fig. 3(a) we show that starting from packing fractions $\eta_{b}=0.1$ and $\eta_{s}=0.01$ and increasing the value of $\eta_{s}$, at first the behavior of the poles seems very similar to that of the mixture with $q=0.5$ : the imaginary part of $\pi_{1}$ increases, while that of $\pi_{2}$ decreases, and eventually there is a crossover at $\eta_{s}^{*}=0.172$ from $\pi_{1}$ to $\pi_{2}$. However, if the value of $\eta_{s}$ is increased further, the imaginary part of $\pi_{2}$, which is then the leading order pole, begins to increase again while at the same time the imaginary part of $\pi_{3}$ decreases sufficiently fast that for $\eta_{s}>0.211 \pi_{3}$ becomes the leading-order pole. Thus for $q=0.35$ and $\eta_{b}=0.1$ there are two crossover points at which the wavelength of oscillations of the asymptotic decay of the correlation functions jumps discontinuously. However, as can be seen in Fig. 3(a), there is only a narrow region of values of $\eta_{s}$ for which $\pi_{2}$ is the leading-order pole. This intermediate crossover behavior disappears once the size ratio becomes slightly smaller. For $q=0.3$ the crossover occurs directly from $\pi_{1}$ to $\pi_{3}$, as is shown in Fig. 3(b) for the same value $\eta_{b}=0.1$. In a similar way the crossover occurs between $\pi_{1}$ and some higher pole as the value of $q$ decreases further. As an example we show in Fig. 4(a) the direct crossover from $\pi_{1}$ to $\pi_{5}$ for $q=0.2$ and fixed $\eta_{b}=0.1$. 

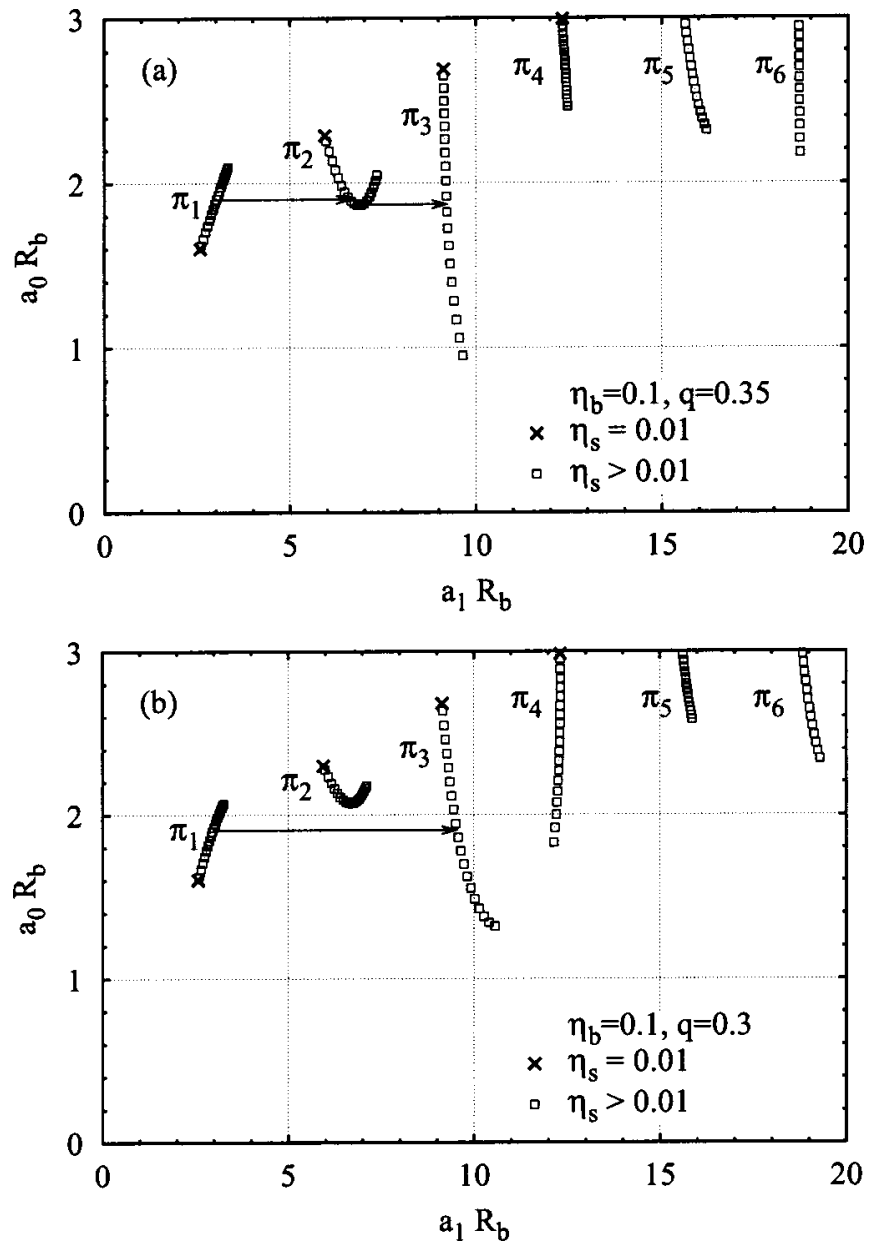

FIG. 3. (a) As in Fig. 2(b) but now for $q=0.35 . \eta_{b}$ is fixed at 0.1 and the crosses refer to $\eta_{s}=0.01$. Increasing $\eta_{s}$ leads first to crossover from $\pi_{1}$ to $\pi_{2}$ at $\eta_{s}=0.172$, left arrow, followed rapidly by a second crossover, at $\eta_{s}$ $=0.211$, from $\pi_{2}$ to $\pi_{3}$. (b) For this size ratio, $q=0.3$, the crossover is directly from $\pi_{1}$ to $\pi_{3}$, see arrow, at $\eta_{s}^{*}=0.189$, where $a_{1}$ increases by a factor of about $1 / q=3.3$.

In order to confirm that the crossover behavior is not peculiar to the PY theory, we repeated the analysis using pair direct correlation functions obtained from the White Bear version of FMT. ${ }^{12,13}$ For weakly asymmetric mixtures at low total packing fractions the agreement between the two approaches is almost perfect. This is not too surprising as the pair direct correlation functions in these limits are very close. For more asymmetric mixtures, say $q=0.2$, and larger packing fractions, quantitative differences between the two theories become visible. This is illustrated in Fig. 4(b). However, the overall agreement between the Percus-Yevick and the White Bear approaches indicates that the general behavior is robust against changes in the details of the approximations; in particular the pattern of crossover is the same for the two approaches.

\section{Crossover lines}

The crossover behavior, exemplified in Figs. 2-4 for various size ratios and for a fixed packing fraction of the big spheres $\eta_{b}=0.1$, can be determined for a range of $\eta_{b}$. In order to represent the locations at which crossover occurs we
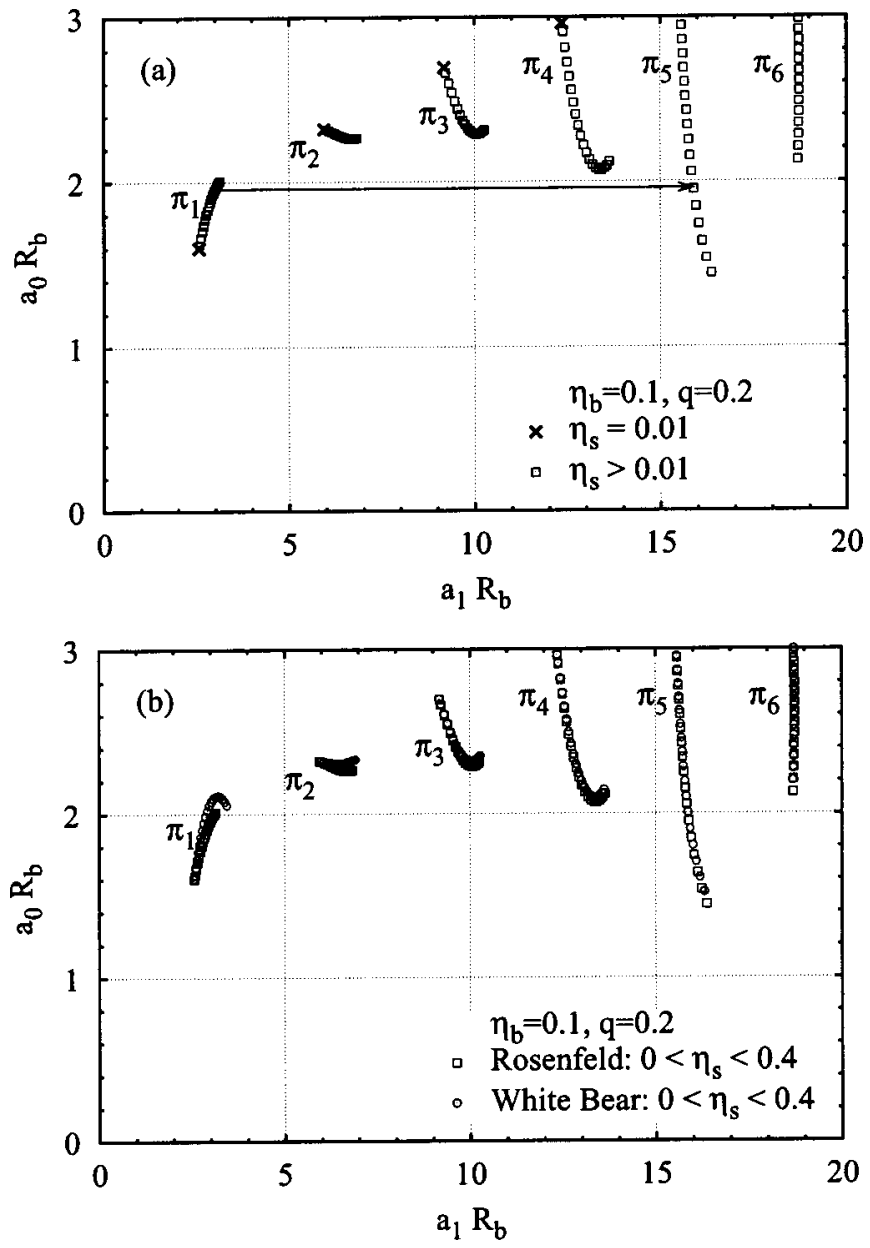

FIG. 4. (a) As in Fig. 2(b) but now for $q=0.2 . \eta_{b}$ is fixed at 0.1 and the crosses refer to $\eta_{s}=0.01$. The imaginary part of $\pi_{1}$ increases with increasing $\eta_{s}$ while that of $\pi_{5}$ decreases so that at $\eta_{s}^{*}=0.278$ crossover occurs, see arrow, from $\pi_{1}$ to $\pi_{5}$ where $a_{1}$ increases by a factor of about $1 / q=5$. (b) Comparison of trajectories from the Rosenfeld (Percus-Yevick) theory (squares) and the White Bear version (circles) for $q=0.2$ and fixed $\eta_{b}$ $=0.1$. Crossover from $\pi_{1}$ to $\pi_{5}$, not shown, occurs for $\eta_{s}^{*}=0.26$ in the White Bear version.

plot a crossover line in the $\left(\eta_{b}, \eta_{s}\right)$ plane for the binary hard-sphere mixture. We start, in Fig. 5(a), by plotting the crossover line for weakly asymmetric mixtures where we find crossover between the $\pi_{1}$ and $\pi_{2}$ poles. For all the size ratios $q$ shown in Fig. 5, the crossover lines are truncated at large total packing fractions. As mentioned earlier, in an attempt to avoid complications of freezing and remain in the stable fluid phase, we deliberately restrict our calculations to state points with $\eta_{s}+\eta_{b}<0.5$.

In the case of very symmetric mixtures, $q \geq 0.7$, the leading-order pole is always $\pi_{1}$, and hence the wave number $a_{1}$ is a continuous function of $\eta_{b}$ and $\eta_{s}$; there is no crossover. For $q \approx 0.65$ we find a short crossover line, as shown in Fig. 5(a), at which $a_{1}$ jumps as the leading-order pole crosses over from $\pi_{1}$ to $\pi_{2}$. It is interesting to note that along a path which connects two points just above and below the crossover line, but which does not intersect the line, the two poles $\pi_{1}$ and $\pi_{2}$ change their identity in a continuous manner. Indeed, the nature of the termination of the crossover line is nontrivial but we do not discuss it here. 

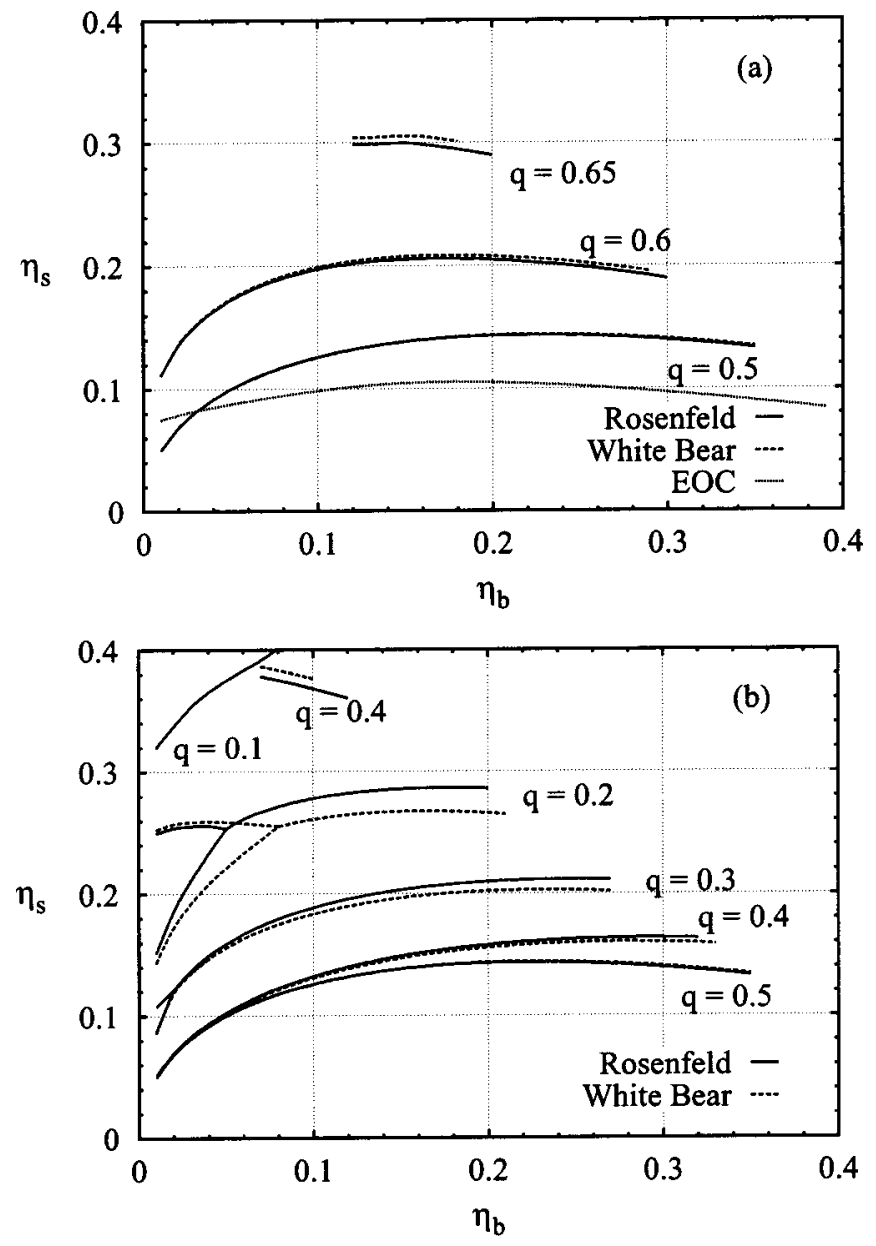

FIG. 5. (a) Crossover lines in the $\left(\eta_{b}, \eta_{s}\right)$ plane for three size ratios $q$ $=0.5,0.6$, and 0.65 . The solid lines denote the results from the Rosenfeld (Percus-Yevick) theory and the dashed lines those from the White Bear version. The results from the two theories are rather close apart from $q$ $=0.65$ where small deviations can be ascertained. For each size ratio the crossover is from $\pi_{1}$ to $\pi_{2}$; below each line the pole $\pi_{1}$ with the smaller $a_{1}$ (larger sphere radius) dominates, whereas above the line $\pi_{2}$ dominates smaller spheres i.e., the smaller spheres. Note that we truncate each line when the total packing fraction is greater than 0.5 . For $q=0.65$ the crossover line is very short; for smaller values of $\eta_{b}$ and $\eta_{s}$ there is no crossover-see text. For $q=0.5$ we also display the crossover line calculated for the effective one-component (EOC) route (dotted line). (b) As in (a) but now for more asymmetric mixtures. For $q=0.3$ the crossover is from $\pi_{1}$ (below the line) to $\pi_{3}$ (above the line), whereas for $q=0.2$ this is from $\pi_{1}$ to $\pi_{5}$. For small values of $\eta_{b}$ crossover can occur via intermediate poles, giving rise to two branches of the lines for $q=0.3$ and 0.2. In the case of $q=0.4$ additional crossover from $\pi_{2}$ to $\pi_{3}$ occurs for values of $\eta_{s}$ $>0.35$. For $q=0.1$ the results from the Rosenfeld theory show crossover from $\pi_{1}$ to $\pi_{10}$ at high values of $\eta_{s}$.

As the size ratio becomes smaller and the mixture more asymmetric, the crossover line rapidly grows in length and spans most of the fluid regime of the phase diagram. At the same time, the crossover occurs at lower values of $\eta_{s}$-see Fig. 5(a). For the three values of $q$ shown in this figure the crossover lines obtained from the Percus-Yevick approximation agree very well with those obtained from the pair direct correlation functions derived from the White Bear version.

For more asymmetric mixtures the crossover behavior can occur via intermediate poles, as shown in Fig. 3(a), which results in a second branch of the crossover line. This second branch appears at low packing fractions of the big spheres, as can be seen in Fig. 5(b) for $q=0.3$ and 0.2. For values of $q<0.5$, the crossover is seen to occur at increasing values of $\eta_{s}$ as $q$ is decreased. In the case $q=0.4$ there is a second crossover from $\pi_{2}$ to $\pi_{3}$ for $\eta_{s}>0.35$.

\section{Pair correlation functions in the neighborhood of the crossover}

The crossover behavior, as described so far, is based on the pole structure of the total correlation functions in complex $k$ (Fourier) space and hence remains quite abstract. In order to demonstrate a clearer physical manifestation of the crossover of the wavelength of the total correlation functions we choose to perform DFT calculations of $h_{i j}(r)$ via the test-particle route. In this calculation one particle of the fluid mixture, either a small or a big one, is fixed at the origin and constitutes the external potential acting on all the other particles of the fluid. By minimizing the grand-canonical potential functional of the hard-sphere mixture in the external field of the fixed particle, $i=s$ or $b$, we obtain the density profiles $\rho_{j}(r)$ and, hence, the pair correlation functions $g_{i j}(r)$ $=\rho_{j}(r) / \rho_{j}$. Recall $h_{i j}(r) \equiv g_{i j}(r)-1$.

If we use the Rosenfeld functional ${ }^{11}$ for $\mathcal{F}_{\text {ex }}\left[\left\{\rho_{i}\right\}\right]$ in the numerical calculation of the total correlation functions, the asymptotic decay length and wavelength of oscillations have the values obtained from the Percus-Yevick pair direct correlation functions, i.e., the values obtained earlier from the poles. ${ }^{17}$ Correspondingly, the asymptotic decay of the total correlation functions obtained from the White Bear version of FMT, using the test-particle route, is that predicted by the White Bear pair direct correlation functions. Using the DFT in the test particle mode, rather than solving the mixture OZ equations (1), has the important advantage that the total correlation functions are more reliable for small separations $r$. Indeed the $h_{i j}(r)$ are usually in good agreement with simulations. Moreover, as emphasized above, they do exhibit asymptotic behavior consistent with the pole analysis. If we were to use the mixture OZ equations (1) to determine $h_{i j}(r)$, the results would be less accurate for small separations when the mixture is highly asymmetric. ${ }^{28}$

Within the context of DFT, the test-particle route to $h_{i j}(r)$ is generally more accurate than the OZ route since the calculation of $\rho_{i}(r)$ via the former requires only $c_{i}^{(1)}(r)$, the one-body direct correlation function, i.e., a first derivative of the functional $\mathcal{F}_{\text {ex }}\left[\left\{\rho_{i}\right\}\right],{ }^{19}$ rather than the second derivative that is employed in the $\mathrm{OZ}$ route.

In Fig. 6 we plot the logarithm of $\left|h_{b b}(r)\right|$ for state points (a) slightly below, (b) on, and (c) slightly above the crossover line for a size ratio $q=0.3$ and a fixed packing fraction of the big spheres, $\eta_{b}=0.15$, calculated for the Rosenfeld functional. The effect of the crossover on the total correlation function is dramatic. While the wavelength of the oscillations in $h_{b b}(r)$ below the crossover line, curve (a), is approximately $\sigma_{b}=2 R_{b}$, the diameter of the big spheres, oscillations develop on the length scale of the small spheres, once $\eta_{s}$ is sufficiently large that we are above the crossover line, curve (c). The change in wavelength is roughly a factor of $q$, i.e., 0.3 in the present case. Moreover, it is striking that this crossover behavior, predicted by the pole analysis and therefore valid strictly for the asymptotic regime $r \rightarrow \infty$, sets 


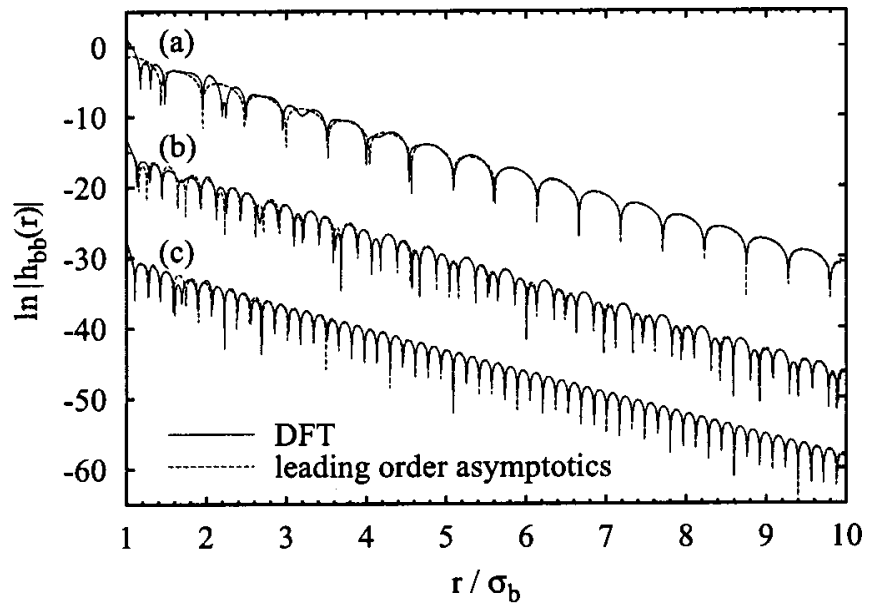

FIG. 6. Plots of $\ln \left|h_{b b}(r)\right|$ for the size ratio $q=0.3$ and fixed $\eta_{b}=0.15$. The solid lines refer to the results of test-particle DFT calculations based on the Rosenfeld functional whereas the dashed lines correspond to leading-order asymptotics - see text. (a) $\eta_{s}=0.15$, (b) $\eta_{s}=0.2028$, the crossover value, (c) $\eta_{s}=0.25$. In (a) and (c) a contribution from a single (dominant) pole [see Eq. (7)] is used in the asymptotic expression whereas in (b) the contributions of two poles are included [see Eq. (18)]. The wavelength of the oscillations changes from about $\sigma_{b}$, the diameter of the big spheres, in (a) to about $0.3 \sigma_{b}$ in (c). Interference effects are clearly visible in (b). The results in (b) and (c) are shifted vertically for clarity of display.

in at intermediate separations. In order to emphasize this point, we show in Fig. 6, alongside the DFT results for $h_{b b}(r)$ (full lines), results based on leading-order asymptotics (dashed lines). The latter were obtained by enforcing the decay described by Eq. (7), with $a_{0}$ and $a_{1}$ given by the calculated leading-order pole. The amplitude $A_{b b}$ and the phase $\Theta_{b b}$ were then fitted to the DFT results at intermediate $r$. Sufficiently far from the crossover line, the agreement between the two sets of results is excellent, except for small separations, $r<2 \sigma_{b}$, where many poles begin to contribute. In the close neighborhood of the crossover line we find, as expected, interference between the oscillatory contributions of two poles. Then one must fit to the form of Eq. (18), i.e., two different amplitudes and two phases were fitted, having calculated independently the values of $a_{0}, \tilde{a}_{0}$ and $a_{1}, \tilde{a}_{1}$. The two-pole fit provides an excellent description of the crossover region; it is not significantly worse than the singlepole fits which apply away from the crossover-see curve (b). Note that the present fitting procedure differs from earlier studies ${ }^{6,8,9}$ of the efficacy of leading-order asymptotics in that those studies calculated amplitudes and phases from the residues, $R_{n}^{i j}$, obtained from the OZ equations (2). Nevertheless, the conclusion that leading-order asymptotics is reliable down to surprisingly small separations $r$ is borne out once again. We should also point out that while we have concentrated on $h_{b b}(r)$, we could equally well have plotted results for the other total correlation functions $h_{b s}(r)$ and $h_{s s}(r)$. Recall that since these functions exhibit asymptotic decay equivalent to that of $h_{b b}(r)$ (only the amplitude and phase differ) we obtain a similar level of agreement between DFT test-particle results and leading-order asymptotics.

From the DFT results it is clear that it is possible to pick up the contribution from the leading-order pole at moderate separations. This implies that features of the crossover be- havior should also be visible in correlation functions obtained by computer simulations, where owing to statistical error one is restricted to small and intermediate separations. Armed with this information, we performed Monte Carlo simulations of the total correlation functions for a binary mixture with $q=0.5$. In order to be able to ascertain a sequence of oscillations in $h_{b b}(r)$ it is important to perform the simulations in a regime of slow exponential decay, i.e., we must remain at high total packing fractions. Thus we fixed $N_{b}=500$, the number of big spheres in the simulation box, which corresponds to $\eta_{b}=0.3$, and increased the number of small spheres from $N_{s}=500$ to 2400 . The results are shown in Fig. 7(a). The crossover behavior is evident in the Monte Carlo results. For the smallest value of $\eta_{s}$ shown $\left(\eta_{s}\right.$ $=0.0375$ ) the wavelength of oscillations is well defined and is approximately $\sigma_{b}$, whereas at the largest value shown $\left(\eta_{s}=0.1753\right)$ the wavelength is roughly $\sigma_{b} / 2$. At intermediate values of $\eta_{s}$ there is clearly interference between two length scales. The results for $h_{b b}(r)$ obtained from DFT are remarkably similar to those from Monte Carlo simulationssee Fig. 7(b). Much of the detail at small and intermediate separations is captured by DFT. Of course, there is no reason to expect the DFT to yield the precise crossover value. The simulations imply that this should occur between $\eta_{s}$ $=0.1125$ and 0.15 . The pole analysis, see Fig. 5, gives a crossover value $\eta_{s}^{*} \approx 0.14$ for $\eta_{b}=0.3$. That crossover clearly apparent in the Monte Carlo simulations is important for two main reasons: (i) in the Monte Carlo simulations no approximations are made for the pair direct correlations functions; one simply calculates $h_{b b}(r)$ directly. (ii) Experimentally determined total correlation functions, for colloidal systems say, also contain statistical noise which makes it difficult to extend results into the true asymptotic regime. The agreement between Monte Carlo simulations and DFT results gives us confidence that we can extract useful information at intermediate separations.

In a second set of simulations the effective onecomponent description was used in order to calculate $h_{b b}(r)$. The depletion potential $W(r)$ which was employed (see Sec. II B) in the simulation is the parametrized form from Ref. 17; we comment on this in the following section. The resulting total correlation functions $h_{b b}(r)$ are shown in Fig. 7(c). These clearly show crossover behavior as $\eta_{s}^{r}$, the packing fraction of the reservoir, is increased. The sequence of the results is similar to that from the simulation of the full binary mixture shown in Fig. 7(a) and to that from the DFT, Fig. 7(b). If the truncated depletion potential from Ref. 29 is employed, which does not include the oscillatory structure in $W(r)$ and, hence, contains only minimal information about correlations between the small spheres, then no crossover in the wavelength of oscillations in the total correlation function $h_{b b}(r)$ is observed-compare the bottom plot in Fig. 7 (c) with the one above it. Note that noise sets in the simulation results for $r / \sigma_{b} \gtrsim 6$.

\section{Highly asymmetric binary mixtures}

We now turn to size ratios $q \leqq 0.2$ for which the full binary mixture treatment, based on calculating the pair direct 

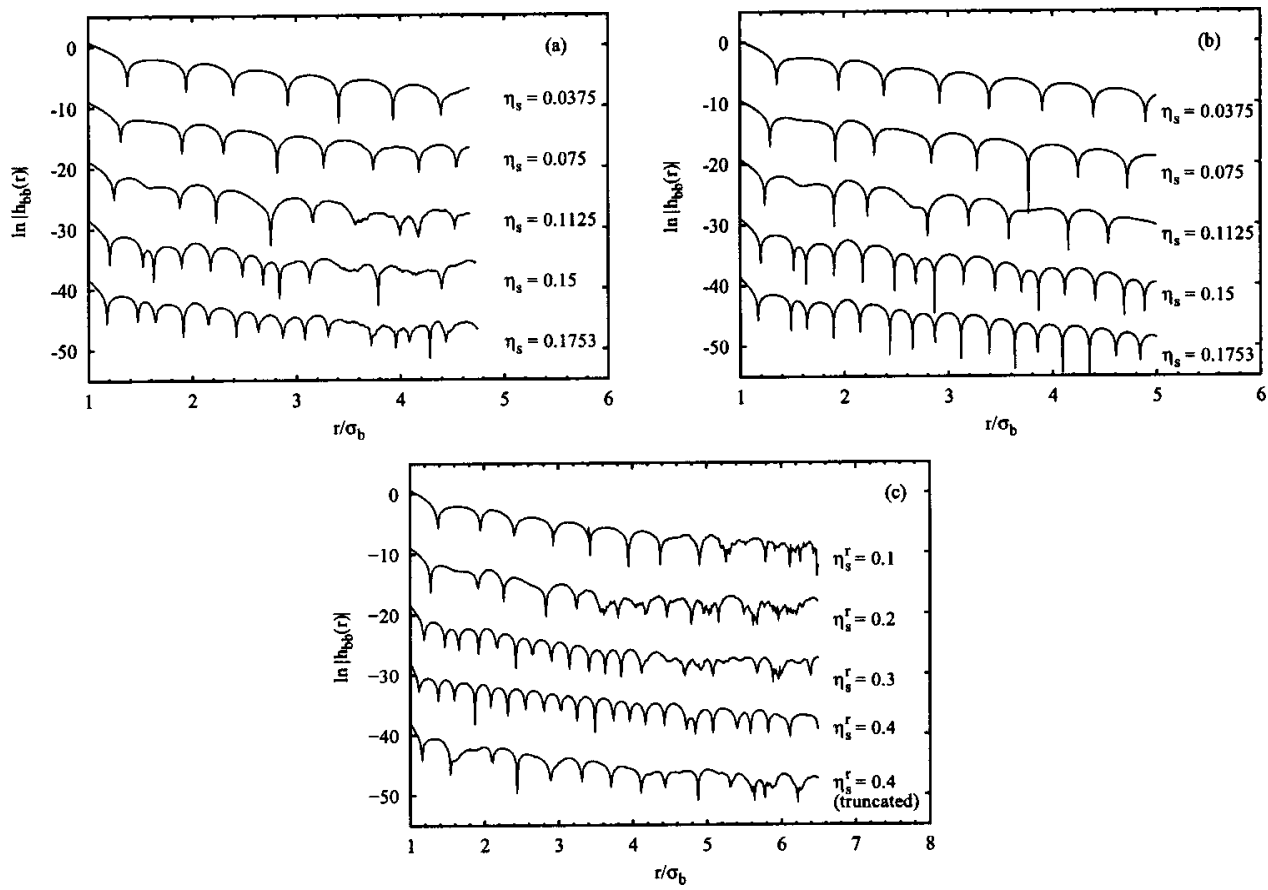

FIG. 7. (a) Results for $\ln \left|h_{b b}(r)\right|$ for a size ratio $q=0.5$ and fixed $\eta_{b}=0.3$ obtained from direct Monte Carlo simulations of the binary mixture. At the two smallest values of $\eta_{s}$ the oscillations have a wavelength of about $\sigma_{b}$, whereas for the largest value of $\eta_{s}$ the wavelength is about $0.5 \sigma_{b}$. For intermediate values of $\eta_{s}$ there is no well-defined wavelength. (b) DFT results using the test-particle procedure and the Rosenfeld functional for the same system as in (a). The results agree remarkably well with those from Monte Carlo simulations. (c) Monte Carlo results for the same system, $q=0.5$ and $\eta_{b}=0.3$, based on an effective one-component description of the mixture using a depletion potential from Ref. 17. $\eta_{s}^{r}$ denotes the packing fraction of the small sphere reservoir. For $\eta_{s}^{r}=0.1$ the oscillations at intermediate $r$ have a well-defined wavelength of about $\sigma_{b}$, whereas for $\eta_{s}^{r}=0.4$ this is reduced to about $0.5 \sigma_{b}$. For intermediate values of $\eta_{s}^{r}$ there is interference. The bottom curve refers to simulation results based on the truncated depletion potential of Götzelmann $e t$ al. (Ref. 29), which does not incorporate properly the correlations of the small spheres. This depletion potential does not yield the crossover that is displayed in the curves above. Note that in each part of the figure the curves are shifted vertically for clarity of display.

correlation functions from PY approximation (or slight modifications thereof), is expected to become less reliable. Rather we treat asymmetric binary mixtures within the framework of the effective one-component picture based on the pair depletion potential $W(r)$-see Sec. II B. This picture is known to become increasingly accurate as $q$ is reduced, i.e., the more asymmetric the mixture becomes. In order to capture details of the asymptotic decay of $h_{b b}(r)$ at arbitrary density $\rho_{b}$, the depletion potential used in this route must itself have the correct asymptotic behavior. Thus, we apply the parameterized form of the depletion potential from Ref. 17. This is constructed, see Eq. (14), so that for a given reservoir density $\rho_{s}^{r}, \lim _{\rho_{b} \rightarrow 0} h_{b b}(r)$ has the correct decay. As remarked earlier, this is not the case for the simplified (truncated) depletion potential of Ref. 29 used in simulation studies of the phase behavior of asymmetric hard-sphere mixtures. ${ }^{15}$ The truncated potential is inadequate for the present purposes.

For size ratios $q \geq 0.25$ we find that the effective onecomponent description results in pole trajectories very similar to those from the binary mixture treatment. The crossover lines found from the two routes are in qualitative agreement; an example is given in Fig. 5(a) for $q=0.5$. It is important to bear in mind that in the former route the size of the small spheres enters only by determining the form and setting the wavelength of oscillations of the depletion potential. That the asymptotic behavior is similar in the two routes, and cross- over occurs in both, is an important success for the effective one-component picture.

We cannot expect quantitative agreement between the two routes for a weakly asymmetric case such as $q=0.5$ since our effective one-component approach omits three and higher-body contributions which should play a role at such size ratios. Note that all the results shown correspond to the Percus-Yevick closure for solving the one-component $\mathrm{OZ}$ equation. The HNC was also employed for a few test cases and similar results were obtained.

If we make the mixture more asymmetric, we find new features. For $q=0.1$, where we expect our effective onecomponent approach to be very reliable, we find that there are qualitative differences between results from the binary mixture treatment and those of the effective one-component fluid. In Fig. 8 we show that for this mixture at fixed $\eta_{b}$ $=0.1$, poles from the two approaches move on similar paths upon increasing the value of $\eta_{s}$ but at very different rates. We do not find the crossover line (at lower $\eta_{b}$ ) from $\pi_{1}$ to $\pi_{10}$, which is shown in Fig. 5(b). Moreover, we find that in the effective one-component picture a purely imaginary pole develops and moves towards the real axis when the reservoir packing fraction of the small spheres, $\eta_{s}^{r}$, is increased. At a value of $\eta_{s}^{r} \approx 0.26$ the purely imaginary pole becomes the leading-order pole, which corresponds to crossover to purely exponential decay of $r h_{b b}(r) ; a_{1}=0$ in Eq. (7). The crossover from an exponentially decaying oscillatory $r h_{b b}(r)$ to a 


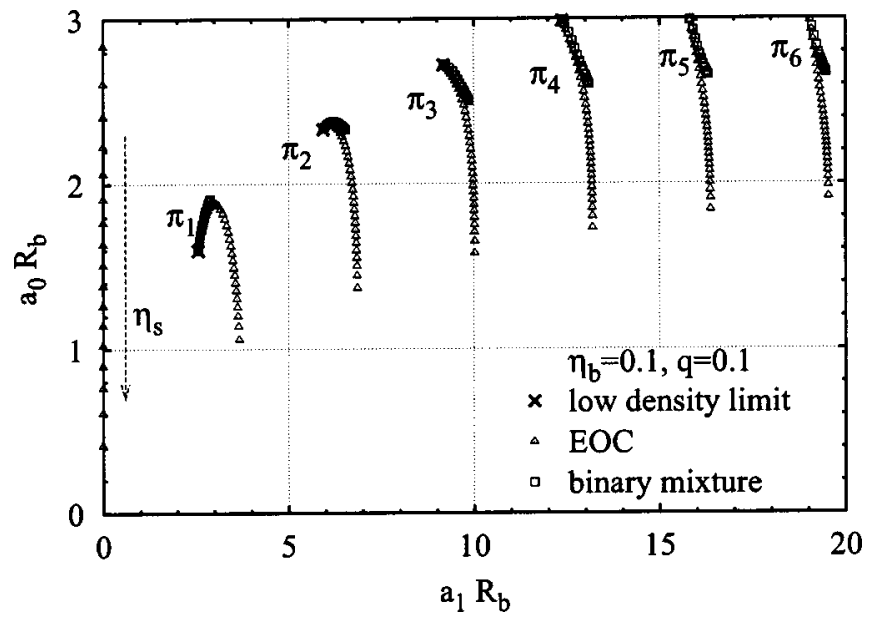

FIG. 8. The trajectories of poles for a binary mixture of hard spheres with size ratio $q=0.1$ and fixed $\eta_{b}=0.1$. The crosses denote the poles evaluated for a very low values of $\eta_{s}$. Upon increasing $\eta_{s}$ the poles calculated from the binary mixture Percus-Yevick route (squares) and from the EOC route (triangles) follow similar paths, but the latter move much more rapidly. Within the EOC there is a pure imaginary pole, $a_{1} \equiv 0$, which moves towards the real axis as the reservoir density $\eta_{s}^{r}$ increases. Near $\eta_{s}^{r}=0.26$ the pure imaginary pole becomes the leading pole, crossover occurs and the ultimate decay of $r h_{b b}(r)$ is purely exponential.

purely exponentially decaying $r h_{b b}(r)$ is well known in other systems where the fluid displays fluid-fluid or liquidvapor phase separation. ${ }^{6-9,30}$ As mentioned in the Introduction, the line in the phase diagram at which such crossover occurs is termed the Fisher-Widom ${ }^{10}$ line, named after the authors who predicted its existence.

As an example of the change of the total correlation function $h_{b b}(r)$ as the Fisher-Widom line is crossed is shown in Fig. 9 for $q=0.05$ and $\eta_{b}=0.1$. Results are plotted for two values of $\eta_{s}^{r}$, one below and the other above the FisherWidom line. $h_{b b}(r)$ is the solution of the PY closure to the effective one-component $\mathrm{OZ}$ equation (15). Again, the asymptotic behavior sets in at intermediate separations, as can be seen from the agreement between $h_{b b}(r)$ (full line) and results obtained by retaining only the leading-order pole contribution (dashed line)—see Fig. 9. For the latter the decay described by Eq. (7) was enforced on the oscillatory side of the Fisher-Widom line, with $a_{0}$ and $a_{1}$ given by the calculated leading-order pole and the amplitude and phase fitted to the numerical results at intermediate $r$. On the monotonic side, where the purely imaginary pole dominates, $a_{0}$ is calculated and only the amplitude is fitted.

A particular feature of the binary hard-sphere mixture is that in the limit $\eta_{b} \rightarrow 0$ the correlation functions must oscillate with a wavelength set by the small spheres-for all $\eta_{s}$ $>0$. This implies that, for sufficiently large $\eta_{s}$, in addition to the Fisher-Widom line which separates an oscillatory region which is rich in big spheres from a monotonic region poor in big spheres, there must be another branch of the FisherWidom line at small values of $\eta_{b}$ separating the aforementioned monotonic region poor in big spheres from an oscillatory region which is even more dilute in big spheres. We find this second branch of the Fisher-Widom line lies at extremely low values of $\eta_{b}$ for $q=0.1$, i.e., $\eta_{b} \approx 10^{-6}$ for intermediate $\eta_{s}^{r}$. Upon decreasing $q$ further this branch of the

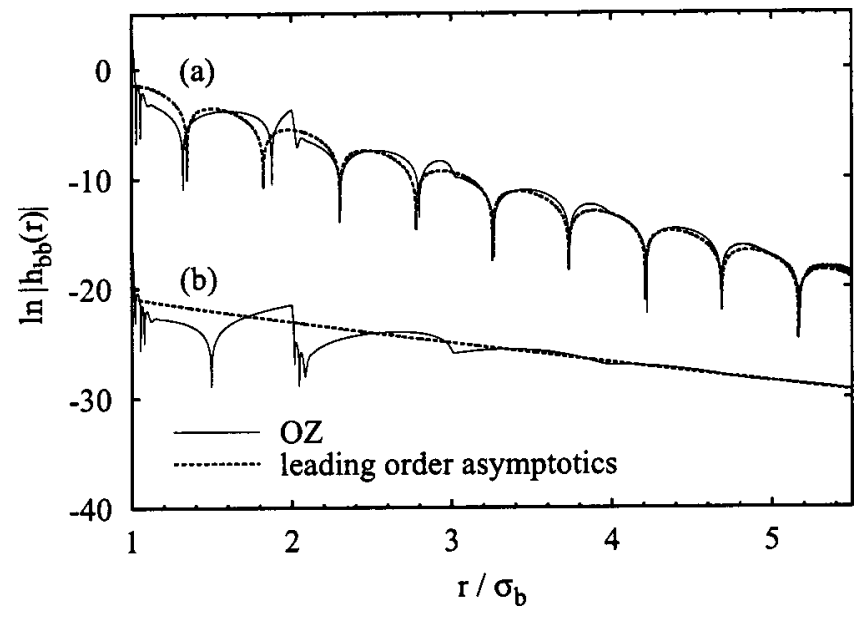

FIG. 9. Plots of $\ln \left|h_{b b}(r)\right|$ for a size ratio $q=0.05$ and fixed $\eta_{b}=0.1$. The solid lines refer to the results obtained from the Percus-Yevick closure of the EOC OZ Eq. (15) based on the depletion potential, whereas the dashed lines correspond to leading-order asymptotics-see text. In (a), $\eta_{s}^{r}=0.12$, a contribution from a single (dominant) complex pole [see Eq. (7)] is used in the asymptotic expression and the wavelength of the oscillations is about $\sigma_{b}$ while in (b), $\eta_{s}^{r}=0.18$, a single pure imaginary pole $\left(a_{1}=0\right)$ contributes and the decay of $h_{b b}(r)$ is monotonic for large $r$. Crossover occurs near $\eta_{s}^{r}$ $=0.15$. The results in (b) are shifted vertically for clarity of display.

Fisher-Widom line rapidly moves closer to the $\eta_{b}=0$ axis. The fact that there are two branches of the Fisher-Widom line is very similar to what is found for a Gaussian core binary mixture. ${ }^{30}$ There one also finds a structural crossover line emerging from the confluence of the two branches. ${ }^{30} \mathrm{We}$ find the equivalent scenario here. The two branches of the Fisher-Widom line meet at some point very close to the $\eta_{b}$ $=0$ axis. Below this point emerges a single crossover line, running close to the axis and terminating at $\eta_{b}=0, \eta_{s}=0$, which separates the two types of oscillatory decay. The Fisher-Widom line is shown in Fig. 10 in the reservoir (a) and the system (b) representations for size ratio $q=0.05$. However, on this scale the left branch of the Fisher-Widom line separating the region in which the pair correlation functions oscillate with a wavelength set by the size of the small spheres from that in which correlations decay monotonically cannot be distinguished from the $\eta_{b}=0$ axis; the line is at even smaller values of $\eta_{b}$ than for $q=0.1$. Nor can one distinguish the structural crossover line which occurs at lower $\eta_{s}$.

A FW line is associated with the presence of a fluid-fluid spinodal. Indeed, the FW line is bounded by the spinodal, which is defined by $a_{0}=0$, i.e., when the pure imaginary pole reaches the origin. ${ }^{7}$ In Fig. 10 we plot the spinodal for $q=0.05$ obtained from the effective one-component (EOC) approach. We did not attempt to calculate the corresponding fluid-fluid binodal. The spinodal and the FW line lie well above the fluid-solid binodal (dotted line) obtained from Monte Carlo simulations of the EOC system. ${ }^{15}$ For $q=0.05$ the fluid-fluid transition is deep in the metastable region of the phase diagram and the accompanying Fisher-Widom line also lies in the metastable region. 

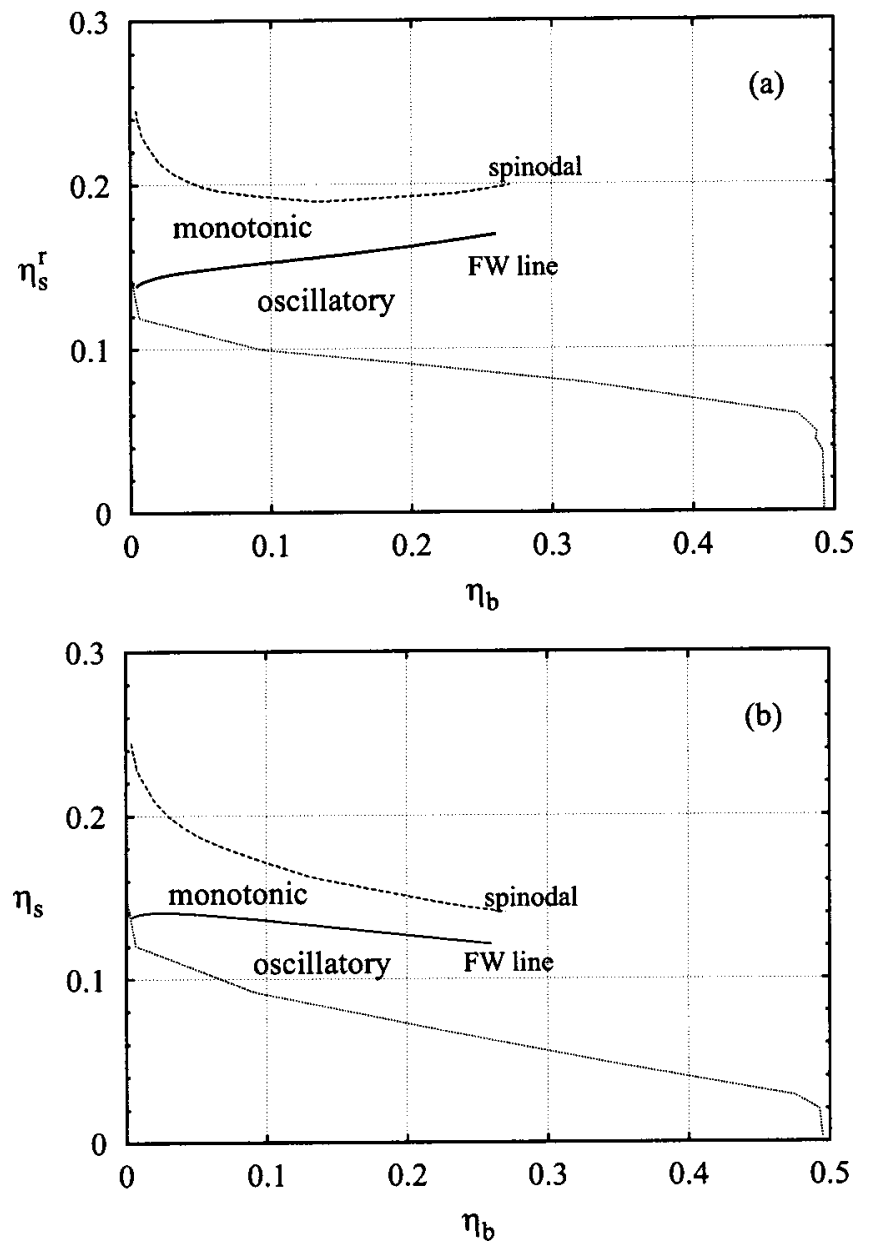

FIG. 10. The (metastable) fluid-fluid spinodal (dashed curve) and the corresponding Fisher-Widom (FW) line (solid curve) separating regimes in which pair correlation functions decay monotonically from those in which they decay in an oscillatory fashion for a binary hard-sphere mixture with $q=0.05$. (a) reservoir representation of the phase diagram; (b) system representation. Note that in the limit $\eta_{b} \rightarrow 0$ the pair correlation functions decay in an oscillatory fashion with a wavelength set by the size of the small spheres. This leads to an additional branch of the FW line for large values of $\eta_{s}$ and a structural crossover line at lower values of $\eta_{s}$. These lines are located at extremely small $\eta_{b}$ so they are not visible on this scale (see text). The dotted line denotes the fluid-solid binodal from Monte Carlo simulations (Ref. 15)

\section{DISCUSSION}

We have carried out extensive studies of the asymptotic, $r \rightarrow \infty$, and intermediate range decay of pair correlation functions in homogeneous binary hard-sphere mixtures. In the case of weakly asymmetric mixtures the full, two-component description is to be preferred whereas for highly asymmetric mixtures (small values of the size ratio $q$ ) the effective onecomponent description is expected to be more accurate. Both descriptions predict the phenomenon of structural crossover, provided $q \leqq 0.7$. At the crossover the wavelength of the longest range oscillation in $h_{i j}(r)$ changes discontinuously from a value set by the diameter of the larger spheres to a value set by the diameter of the smaller species. The crossover occurs when the leading-order pole of the Fourier transform, $\hat{h}_{i j}(k)$, changes from one branch to another. For certain size ratios the change does not take place in a single step but can occur via crossover from one branch of poles to a branch with an intermediate wavelength followed by further crossover to a shorter wavelength branch-see Fig. 3(a) for $q=0.35$. To the best of our knowledge, this is the first time structural crossover has been predicted for hard-sphere mixtures. ${ }^{31}$ The crossover is robust; it occurs in both the Rosenfeld and White Bear version of DFT as well as in a completely different treatment (integral equation treatment) of the effective one-component model. Moreover, the crossover lines in the $\left(\eta_{b}, \eta_{s}\right)$ plane are calculated to be quite close in the different theories. Were the crossover to manifest itself only at longest range (asymptotically) it would not be of much practical interest. However, our DFT studies and Monte Carlo simulations demonstrate that crossover manifests itself at intermediate as well as at longest range-see Figs. 6 and 7. One can observe different characteristic wavelengths on opposite sides of the crossover line. Close to crossover there are interference effects. Leading-order asymptotics, based on the leading-order complex poles, account accurately for the intermediate range behavior of $h_{i j}(r)$. Note that throughout we have focused on $h_{b b}(r)$, the big-big correlation function, but as emphasized earlier, the other correlation functions $h_{b s}(r)$ and $h_{s s}(r)$ will display equivalent features of crossover behavior.

The EOC description performs remarkably well for size ratios where one might expect the many-body contributions omitted in the effective pair potential treatment might be significant. Thus, for $q=0.5$ the EOC yields a reasonable crossover line [Fig. 5(a)] and a reliable account of the behavior of $h_{b b}(r)$ at intermediate values of $r$-see the simulation results of Fig. 7. It is important to recognize that the EOC provides a valid account of crossover provided an accurate approximation is employed for the depletion potential $W(r)$. The latter must incorporate the correct asymptotic decay, i.e., the oscillations which are determined by correlations in the reservoir of small spheres. If these are not properly incorporated, the EOC picture fails to describe crossover-see Fig. 7 (c). In the case of highly asymmetric mixtures, $q \leqq 0.1$, where the EOC description should be accurate, we find a Fisher-Widom line with two branches. This line is intimately connected with the existence of a fluid-fluid spinodal within the EOC analysis—see Fig. 10 for $q=0.05$. The presence of the spinodal ensures that there must be a region of monotonic decay of pair correlations, i.e., there must be OrnsteinZernike behavior in the neighborhood of the critical point. What is special about the present system is that correlations must decay in a damped oscillatory fashion along the axis $\eta_{s}=0$ (the pure fluid of small hard spheres), and this is responsible for the two branches at large values of $\eta_{s}$ (or $\eta_{s}^{r}$ ). At lower values of $\eta_{s}$ (or $\eta_{s}^{r}$ ) one finds the same type of structural crossover line as that ascertained in Ref. 30 for the Gaussian core mixture. Indeed the overall scenario we find for small values of $q$ mimics, to a large extent, that found for certain Gaussian core mixtures exhibiting fluid-fluid phase separation. ${ }^{30}$ The main difference is that the fluid-fluid phase transition and the accompanying Fisher-Widom line is metastable with respect to the fluid-solid transition in the present hard-sphere mixture case. If one could suppress freezing and perform measurements in the metastable region (see Fig. 10) then one would observe interesting crossover from damped 
oscillatory asymptotic decay of correlations to monotonic decay as $\eta_{s}$ is increased at fixed $\eta_{b}$, i.e., as the total packing fraction is increased. Such behavior is somewhat counterintuitive and would provide a signature of the (incipient) fluidfluid spinodal.

A similar situation exists for the extreme nonadditive hard-sphere mixture, namely, the Asakura-Oosawa-Vrij model of a colloid-polymer mixture which treats the colloids as hard spheres and the polymer coils as ideal interpenetrating spheres that are excluded from colloids. A recent DFT treatment $^{32}$ determined the fluid-fluid spinodal and binodal and the associated Fisher-Widom line for various polymer to colloid size ratios $q$. Increasing $\eta_{p}^{r}$, the polymer reservoir packing fraction, leads generally to crossover from oscillatory to exponential decay, and for sufficiently large values of $q$ this can occur in a stable (fluid) region of the phase diagram. ${ }^{32}$ The important difference between the results in Ref. 32 and the present ones is that for the latter pair correlations must exhibit oscillatory decay as $\eta_{b} \rightarrow 0$, whereas in the Asakura-Oosawa-Vrij model the smaller species is replaced by ideal, interpenetrating spheres for which the pair correlation function decays exponentially, i.e., the decay for $\eta_{c}$ (equivalent to $\eta_{b}$ ) $\rightarrow 0$ is monotonic rather than oscillatory. This means that the Fisher-Widom line does not have two branches, as is found in the present case, and there is no crossover line at small $\eta_{p}^{r}$. ${ }^{32}$

Although we have focused here on crossover behavior for the pair correlation functions in bulk binary hard-sphere mixtures, it is important to recognize that similar features can be found for the decay of correlations in inhomogeneous fluid mixtures. The one-body density profiles of a hardsphere mixture close to a wall that exhibits short-ranged wall-fluid interactions, the solvation force for the same mixture confined between two walls and the depletion potential between two big (colloidal) particles immersed in a binary mixture of smaller hard spheres will exhibit equivalent features determined by the same physical considerations, i.e., by the pole structure described in the present paper. We shall return to this topic in a later paper.

In real fluids, whether these are mixtures of simple (atomic) components or of two types of (spherical) colloidal particles, the interparticle potentials are not precisely those of hard spheres. The potential function is never infinitely repulsive. It is important to enquire how our results might change for repulsive interparticle potentials that are softer than hard spheres. Provided the interparticle potentials remain short ranged (no power-law decay), the pole structure of the $\hat{h}_{i j}(k)$ should be equivalent to that described here, and all $h_{i j}(r)$ must decay in the same fashion as $r \rightarrow \infty$. Thus, in the oscillatory regime the mixture must find a unique wavelength for the longest ranged oscillations. It follows that, provided the effective diameters are sufficiently different, the mixture should display a structural crossover for some choice of thermodynamic parameters. Given that structural crossover occurs in the binary Gaussian core mixture, ${ }^{30}$ where the pair potentials are very soft, it is difficult to imagine that the phenomenon would disappear for mixtures that are much more harshly repulsive.

Since we have demonstrated that the characteristic wavelength of the oscillations in $g_{i j}(r)$ manifests itself at moderate separations, even when statistical errors are present, we believe that it should be possible to observe the phenomenon of crossover in binary mixtures of colloidal particles using real space techniques of the type employed already for one-component systems. ${ }^{3,4}$

\section{ACKNOWLEDGMENTS}

We have benefited from conversations with A. J. Archer and R. van Roij. R.E. and M.D. are grateful to S. Dietrich and his colleagues for their hospitality, and R.E. thanks the Alexander von Humboldt Foundation for support under GRO/1072637 during his stay at the MPI in Stuttgart.

\section{APPENDIX: PAIR DIRECT CORRELATION FUNCTIONS WITHIN FMT}

The weight functions within the FMT approach, which describe the fundamental geometrical measures of a sphere of component $i$ of radius $R_{i}$, are

$$
\begin{aligned}
& w_{3}^{i}(\mathbf{r})=\Theta\left(R_{i}-|\mathbf{r}|\right), \\
& w_{2}^{i}(\mathbf{r})=\delta\left(R_{i}-|\mathbf{r}|\right), \\
& w_{1}^{i}(\mathbf{r})=\frac{1}{4 \pi R_{i}} w_{2}^{i}(\mathbf{r}), \\
& w_{0}^{i}(\mathbf{r})=\frac{1}{4 \pi R_{i}^{2}} w_{2}^{i}(\mathbf{r}), \\
& \mathbf{w}_{2}^{i}(\mathbf{r})=\frac{\mathbf{r}}{|\mathbf{r}|} \delta\left(R_{i}-|\mathbf{r}|\right), \\
& \mathbf{w}_{1}^{i}(\mathbf{r})=\frac{1}{4 \pi R_{i}} \mathbf{w}_{2}^{i}(\mathbf{r}),
\end{aligned}
$$

where $\delta(r)$ denotes the Dirac $\delta$ function and $\Theta(r)$ denotes the Heaviside function. The convolution product $\otimes$ used in Eq. (11) is defined as

$$
w_{\alpha}^{i} \otimes w_{\beta}^{j} \equiv \int d \mathbf{r} w_{(\alpha)}^{i}\left(\mathbf{r}_{i}-\mathbf{r}\right) w_{\beta}^{j}\left(\mathbf{r}_{j}-\mathbf{r}\right) .
$$

The spheres do not overlap for separations $r>R_{i}+R_{j}$, with $i, j=b, s$ and $r=\left|\mathbf{r}_{i}-\mathbf{r}_{j}\right|$. This leads to $w_{\alpha}^{i} \otimes w_{\beta}^{j}=0$. Therefore, we obtain $c_{i j}^{(2)}\left(r>R_{i}+R_{j}\right)=0$, which is a key feature for all pair direct correlation functions based on FMT.

In the case $\left|R_{i}-R_{j}\right|<r<R_{i}+R_{j}$ the surfaces of the spheres intersect and we obtain the results

$$
\begin{aligned}
w_{3}^{i} \otimes w_{3}^{j}= & \frac{\pi}{3}\left(-\frac{3}{2} R_{i}^{2} r-\frac{3}{4} \frac{R_{i}^{4}}{r}+2 R_{i}^{3}+\frac{1}{4} r^{3}+\frac{3}{2} \frac{R_{i}^{2} R_{j}^{2}}{r}\right. \\
& \left.-\frac{3}{2} R_{j}^{2} r-\frac{3}{4} \frac{R_{j}^{4}}{r}+2 R_{j}^{3}\right) \\
w_{2}^{i} \otimes w_{3}^{j}= & 2 \pi R_{i}\left(R_{i}+\frac{1}{2} \frac{R_{j}^{2}-r^{2}-R_{i}^{2}}{r}\right) \\
w_{3}^{i} \otimes w_{2}^{j}= & 2 \pi R_{j}\left(R_{j}+\frac{1}{2} \frac{R_{i}^{2}-r^{2}-R_{j}^{2}}{r}\right)
\end{aligned}
$$




$$
\begin{aligned}
w_{2}^{i} \otimes w_{2}^{j} & =\frac{2 \pi R_{i} R_{j}}{r}, \\
\mathbf{w}_{2}^{i} \otimes \mathbf{w}_{2}^{j} & =\pi\left(\frac{R_{i}^{2}+R_{j}^{2}}{r}-r\right),
\end{aligned}
$$

which simplify considerably when $R_{i}=R_{j}$. For spheres with different radii, it is possible that a small sphere is completely contained within a big sphere, and for $r<R_{b}-R_{s}$ we obtain

$$
\begin{aligned}
& w_{3}^{i} \otimes w_{3}^{j}=\frac{4 \pi}{3} R_{s}^{3}, \\
& w_{2}^{b} \otimes w_{3}^{s}=w_{3}^{s} \otimes w_{2}^{b}=0, \\
& w_{3}^{b} \otimes w_{2}^{s}=w_{2}^{s} \otimes w_{3}^{b}=4 \pi R_{s}^{2}, \\
& w_{2}^{i} \otimes w_{2}^{j}=\mathbf{w}_{2}^{i} \otimes \mathbf{w}_{2}^{j}=0 .
\end{aligned}
$$

Taking into account that the vectorlike weighted densities vanish in the bulk limit, then $\partial^{2} \Phi\left(\left\{n_{\nu}\right\}\right) / \partial n_{\alpha} \partial \mathbf{n}_{\beta}=0$ in both the Rosenfeld and the White Bear versions of the functional and mixed scalarlike and vectorlike convolutions in Eq. (11) are not required. The remaining convolutions are proportional to those given above in Eqs. (A8)-(A16).

The second partial derivatives of $\Phi$ with respect to the weighted densities $n_{\alpha}, \chi_{\mathrm{RF}}^{(i)} \equiv \partial^{2} \Phi^{\mathrm{RF}} / \partial n_{3} \partial n_{i}$, required in Eq. (11), are for the case of the Rosenfeld functional:

$$
\begin{aligned}
& \chi_{\mathrm{RF}}^{(0)}=\frac{1}{1-n_{3}}, \\
& \chi_{\mathrm{RF}}^{(1)}=\frac{n_{2}}{\left(1-n_{3}\right)^{2}}, \\
& \chi_{\mathrm{RF}}^{(2)}=\frac{n_{1}}{\left(1-n_{3}\right)^{2}}+\frac{n_{2}^{2}}{4 \pi\left(1-n_{3}\right)^{3}}, \\
& \chi_{\mathrm{RF}}^{(3)}=\frac{n_{0}}{\left(1-n_{3}\right)^{2}}+2 \frac{n_{1} n_{2}}{\left(1-n_{3}\right)^{3}}+\frac{n_{2}^{3}}{4 \pi\left(1-n_{3}\right)^{4}} .
\end{aligned}
$$

In the case of the White Bear version of the functional the second partial derivatives $\chi_{\mathrm{WB}}^{(i)} \equiv \partial^{2} \Phi^{\mathrm{WB}} / \partial n_{3} \partial n_{i}$ are given by

$$
\begin{aligned}
\chi_{\mathrm{WB}}^{(0)}= & \chi_{\mathrm{RF}}^{(0)}, \\
\chi_{\mathrm{WB}}^{(1)}= & \chi_{\mathrm{RF}}^{(1)}, \\
\chi_{\mathrm{WB}}^{(2)}= & \chi_{\mathrm{RF}}^{(2)} \\
& +\frac{n_{2}^{2}\left[n_{3}\left(5 n_{3}-n_{3}^{2}-2\right)-2\left(1-n_{3}\right)^{3} \ln \left(1-n_{3}\right)\right]}{12 \pi\left(1-n_{3}\right)^{3} n_{3}^{3}},
\end{aligned}
$$

$$
\chi_{\mathrm{WB}}^{(3)}=\chi_{\mathrm{RF}}^{(3)}+\frac{n_{2}^{3}\left[6\left(1-n_{3}\right)^{4} \ln \left(1-n_{3}\right)-n_{3}\left(21 n_{3}-26 n_{3}^{2}+14 n_{3}^{3}-6\right)\right]}{36 \pi\left(1-n_{3}\right)^{4} n_{3}^{4}} .
$$

Owing to the new form of the functional an additional term arises in the sum (11),

$$
\chi_{\mathrm{WB}}^{(22)} \equiv \frac{\partial^{2} \Phi^{\mathrm{WB}}}{\partial n_{2} \partial n_{2}}=\chi_{\mathrm{RF}}^{(1)} \frac{n_{3}+\left(1-n_{3}\right)^{2} \ln \left(1-n_{3}\right)}{6 \pi n_{3}^{2}} .
$$

${ }^{1}$ J.-P. Hansen and I. R. McDonald, Theory of Simple Liquids, 2nd ed. (Academic, London, 1986).

${ }^{2}$ P. A. Egelstaff, An Introduction to the Liquid State, 2nd ed. (Clarendon, Oxford, 1992).

${ }^{3}$ M. Brunner, C. Bechinger, W. Strepp, V. Lobaskin, and H. H. von Grünberg, Europhys. Lett. 58, 926 (2002).

${ }^{4}$ R. Klein, H. H. von Grünberg, C. Bechinger, M. Brunner, and V. Lobaskin, J. Phys.: Condens. Matter 14, 7631 (2002).

${ }^{5}$ J. L. Lebowitz and J. K. Percus, J. Math. Phys. 4, 248 (1963).

${ }^{6}$ R. Evans, R. J. F. Leote de Carvalho, J. R. Henderson, and D. C. Hoyle, J. Chem. Phys. 100, 591 (1994). See also G. A. Martynov, Fundamental Theory of Liquids: Method of Distribution Functions (Adam Hilger, Bristol, 1992).

${ }^{7}$ R. Evans, J. R. Henderson, D. C. Hoyle, A. O. Parry, and Z. A. Sabeur, Mol. Phys. 80, 755 (1993).

${ }^{8}$ R. J. F. Leote de Carvalho, R. Evans, D. C. Hoyle, and J. R. Henderson, J. Phys.: Condens. Matter 6, 9275 (1994).

${ }^{9}$ M. Dijkstra and R. Evans, J. Chem. Phys. 112, 1449 (2000).

${ }^{10}$ M. E. Fisher and B. Widom, J. Chem. Phys. 50, 3756 (1969).

${ }^{11}$ Y. Rosenfeld, Phys. Rev. Lett. 63, 980 (1989); see also Y. Rosenfeld, D. Levesque, and J.-J. Weis, J. Chem. Phys. 92, 6818 (1990).

${ }^{12}$ R. Roth, R. Evans, A. Lang, and G. Kahl, J. Phys.: Condens. Matter 14, 12063 (2002).
${ }^{13}$ Y.-X. Yu and J. Wu, J. Chem. Phys. 117, 10165 (2002).

${ }^{14}$ J. L. Lebowitz, Phys. Rev. 133, A895 (1964).

${ }^{15}$ M. Dijkstra, R. van Roij, and R. Evans, Phys. Rev. Lett. 81, 2268 (1998); 82, 117 (1999); Phys. Rev. E 59, 5744 (1999).

${ }^{16}$ J. L. Lebowitz and J. S. Rowlinson, J. Chem. Phys. 41, 133 (1964)

${ }^{17}$ R. Roth, R. Evans, and S. Dietrich, Phys. Rev. E 62, 5360 (2000).

${ }^{18}$ D. Goulding and S. Melchionna, Phys. Rev. E 64, 011403 (2001).

${ }^{19}$ R. Evans, Adv. Phys. 28, 143 (1979).

${ }^{20}$ G. A. Mansoori, N. F. Carnahan, K. E. Starling, and T. W. Leland, Jr., J. Chem. Phys. 54, 1523 (1971).

${ }^{21}$ N. F. Carnahan and K. E. Starling, J. Chem. Phys. 51, 635 (1969).

${ }^{22}$ R. Roth, R. Evans, and A. A. Louis, Phys. Rev. E 64, 051202 (2001).

${ }^{23}$ B. Götzelmann, R. Roth, S. Dietrich, M. Dijkstra, and R. Evans, Europhys. Lett. 47, 398 (1999).

${ }^{24}$ P. Perry and G. J. Throop, J. Chem. Phys. 57, 1827 (1972).

${ }^{25}$ J. R. Henderson and Z. A. Sabeur, J. Chem. Phys. 97, 6750 (1992); Mol. Phys. 82, 765 (1994).

${ }^{26}$ Y. Rosenfeld, Phys. Rev. A 33, 2025 (1986); R. J. F. Leote de Carvalho, R. Evans, and Y. Rosenfeld, Phys. Rev. E 59, 1435 (1999).

${ }^{27}$ W. G. Hoover and F. H. Ree, J. Chem. Phys. 49, 3609 (1968).

${ }^{28}$ See the discussion in Sec. 3.6 of Ref. 32 which describes similar failings for the OZ route in the Asakura-Oosawa-Vrij model of colloid-polymer mixtures.

${ }^{29}$ B. Götzelmann, R. Evans, and R. Dietrich, Phys. Rev. E 57, 6785 (1998).

${ }^{30}$ A. J. Archer and R. Evans, Phys. Rev. E 64, 041501 (2001).

${ }^{31}$ G. Kahl and G. Pastore, J. Phys. A 24, 2995 (1991) computed two series of poles of the Laplace transform of $h_{i j}(r)$ for a binary hard-sphere mixture treated in Percus-Yevick approximation, but did not discuss crossover; neither did C. Tutschka and G. Kahl, Phys. Rev. E 65, 051104 (2002).

${ }^{32}$ M. Schmidt, H. Löwen, J. M. Brader, and R. Evans, J. Phys.: Condens. Matter 14, 9353 (2002) 